\title{
La investigación de las arquitecturas y paisajes monumentales del neolítico en el noroeste peninsular: Una aproximación bibliométrica
}

\section{The research history of Neolithic monumental architectures and landscapes in northwest Iberia: a bibliometric approach}

\author{
Elías LóPEZ-RoMERo \\ Instituto de Ciencias del Patrimonio (Incipit-CSIC) \\ San Roque 2.15704 Santiago de Compostela \\ lopezelias@hotmail.com
}

Recibido: 15-12-2012

Aceptado: 11-03-2013

\begin{abstract}
RESUMEN
El presente trabajo tiene por objetivo analizar el modo en que la monumentalidad prehistórica ha sido abordada en el noroeste de la Península Ibérica. Para ello, se han utilizado criterios bibliométricos de análisis. Se estudia de forma conjunta la evidencia publicada para Galicia y Norte de Portugal (territorios al norte del Duero); el área occidental de Asturias ha sido igualmente tenida en cuenta cuando la discusión de los trabajos se enmarcaba explícitamente en los procesos del noroeste, algo que sin embargo es muy poco frecuente. El rango cronológico de la muestra comprende desde finales del siglo XIX (1888) hasta 2010. Se analizan cuestiones como los temas preferentes de investigación, las regiones más intensamente estudiadas o el grado de colaboración e internacionalización de la investigación. Se plantea una sintesis interpretativa sobre los resultados y se ofrece una lectura sobre las tendencias más actuales en la materia y sus posibles lineas de desarrollo.
\end{abstract}

Palabras Clave: Neolitico. Megalitismo. Península Ibérica. Galicia. Norte de Portugal. Bibliometría.

\begin{abstract}
This paper aims at analysing the way the prehistoric monuments in the northwest of the Iberian Peninsula have been studied. A bibliometric approach has been set up for this purpose. The paper jointly considers the evidence for Galicia and north of Portugal (regions north to the Douro); the western area of Asturias has also been taken into account when the research context of the papers explicitly dealt with the Iberian northwest. The sample date range spans from the late 19th century (1888) to 2010. The thematic scope of the papers, the most studied regions or the degree of collaboration and internationalisation of research are some of the variables under study. Finally, an interpretative synthesis on the results, on the current trends and on the research perspectives for the region is provided.
\end{abstract}

KEY worDs: Neolithic. Megalithic monuments. Iberian Peninsula. Galicia. Northern Portugal. Bibliometry. 


\section{Introducción}

El estudio de la monumentalidad en el noroeste de la Península Ibérica tiene una larga tradición. Los investigadores portugueses y españoles se han interesado desde una época muy temprana por las manifestaciones arquitectónicas que, de forma a menudo conspicua, forman parte del paisaje de la región. En este contexto el presente estudio tiene por objetivo ofrecer una visión global del modo en que la monumentalidad prehistórica ha sido abordada en la región (fig. 1). ¿Cuáles han sido las tendencias en la investigación a lo largo del tiempo? ¿Qué grado de internacionalización presenta la investigación del fenómeno en la zona? ¿Cuál es el nivel de colaboración entre los distintos autores? ¿Cuál es el peso de las Universidades y de los centros de investigación en el desarrollo de los estudios sobre monumentalidad? ¿Qué regiones han sido analizadas con mayor intensidad? ¿Cuáles son las propuestas actuales en la materia? Como complemento de las lecturas epistemológicas, teóricas, bibliográficas y arqueográficas, el artículo plantea una aproximación cuantitativa por medio de un análisis bibliométrico. Este tipo de análisis es relativamente frecuente en el estudio de la Prehistoria peninsular, donde se observan dos grandes tendencias. Por un lado, varios trabajos se han planteado como método de evaluación de las revistas y del seguimiento de los criterios de indexación (e.g. Rodríguez Alcalde et al. 1993, 1996; García Marín et al. 1997; Cruells 1995; ArmadaPita 2009); en el caso de las publicaciones con mayor trayectoria este tipo de aproximación ha permitido, además, ofrecer un panorama amplio sobre las tendencias generales de investigación. Por otro lado, se han utilizado como un método eficaz para valorar el estado y evolución de un campo concreto de investigación (e.g. García-Heras 1997; Cruz Berrocal et al. 1999; Vilaseco Vázquez 2001; Cordero et al. 2006; López-Romero y Montero Ruiz 2006; Montero Ruiz et al. 2007; Palomar et al. 2009). Los estudios relativos al ámbito de la Arqueometría predominan en este segundo bloque. Para Galicia contamos con dos trabajos que han abordado el estudio de las publicaciones sobre Prehistoria y Arqueología (Abad Vidal et al. 2000) y las tendencias en la excavación de los monumentos (Vilaseco Vázquez 2001) siguiendo criterios bibliométricos.

En un intento por comprender de forma integrada las dinámicas de investigación, se analiza de forma

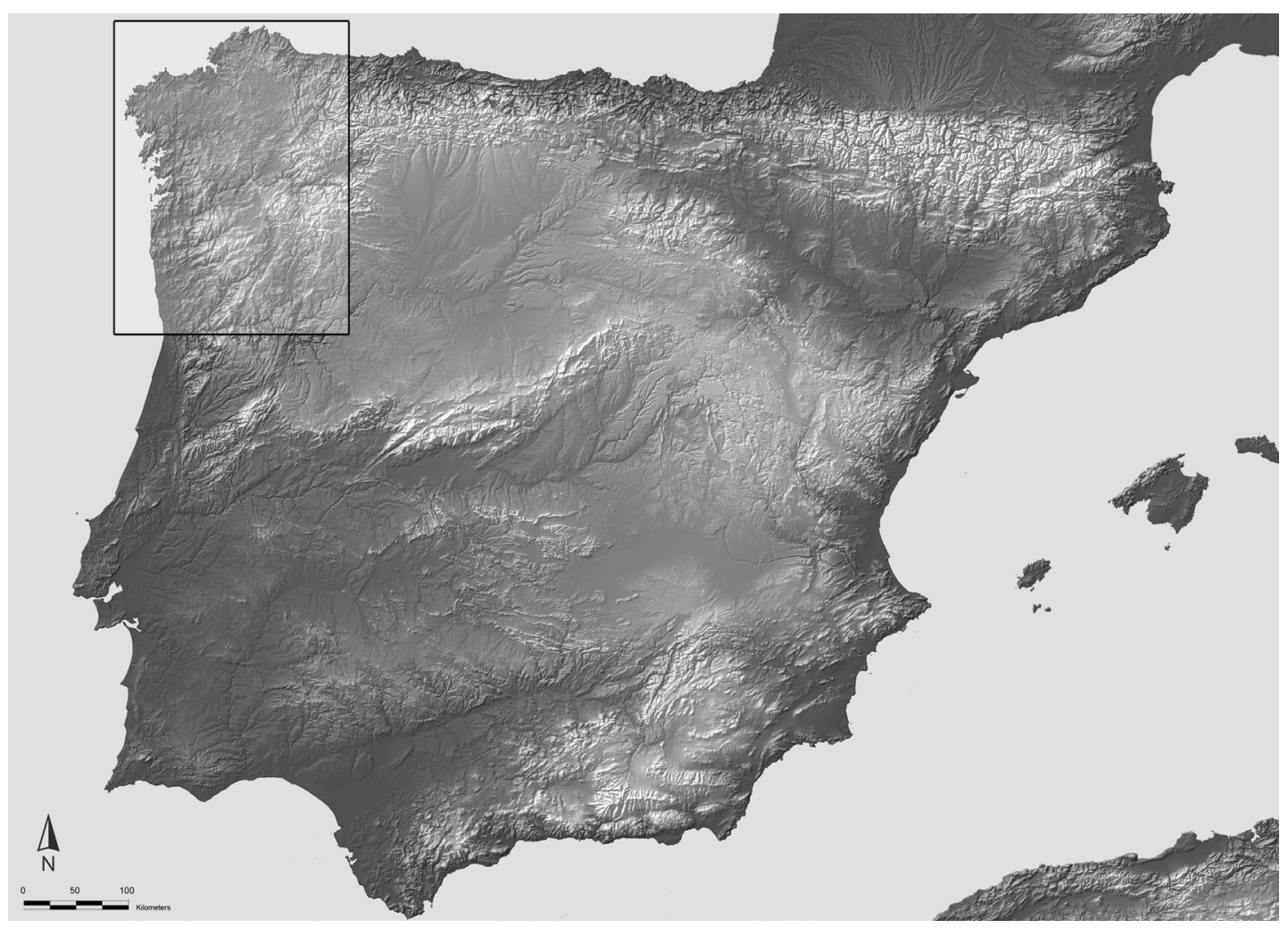

Fig 1.- La región de estudio en el marco general de la Península Ibérica. 
conjunta la evidencia para Galicia y Norte de Portugal (territorios al norte del Duero). El área occidental de Asturias ha sido igualmente tenida en cuenta cuando la discusión de los trabajos se enmarcaba explícitamente en los procesos del noroeste, algo que sin embargo ocurre en muy pocas ocasiones.

El artículo se organiza en tres secciones principales. En la primera se exponen la metodología seguida, los criterios de selección de la muestra y sus características. A continuación se procede al análisis de la muestra a través de una serie de variables consideradas relevantes para responder a los interrogantes planteados. Por último se lleva a cabo una síntesis interpretativa de los resultados, y se ofrece una lectura sobre las tendencias y líneas de desarrollo de los estudios sobre monumentalidad en el noroeste.

\section{Metodología y naturaleza de la muestra}

El trabajo ha constado de cuatro fases. En un primer momento se elaboró un listado de 83 publicaciones susceptibles de contener información sobre el fenómeno en la región. Se utilizaron únicamente revistas, series monográficas y actas publicadas de congresos, quedando excluídas las monografías y los informes técnicos. Las publicaciones son de orientación regional, nacional, peninsular e internacional dedicadas a la Prehistoria, Arqueología, Neolítico y megalitismo tanto de instituciones ubicadas en la Península Ibérica como fuera de ella. No se estableció ningún sesgo por idioma de publicación, periodicidad o ránking. Las revistas y series monográficas proporcionan por sus características una información variada y con múltiples posibilidades de comparación (García Marín et al. 1997: 51-2). Los congresos científicos juegan un papel importante en la comunicación e intercambio, a pesar del escaso valor que se les ha dado en algunos análisis bibliométricos. Para el análisis de los congresos se ha tenido en cuenta la fecha de publicación, no la de celebración.

En segundo lugar se definieron las variables a analizar y se creo una estructura de base de datos relacional (MS Access) para el vaciado de la información. A continuación se llevó a cabo el vaciado propiamente dicho, y se introdujo la información en la base de datos. Por último, y por medio de consultas a la base de datos, se analizó la información con ayuda del paquete estadístico SPSS (v.19).

Las variables objeto de estudio se han agrupado en cuatro bloques:

a) Cronología y morfología: año de publicación; nombre de la revista/congreso; tipo de contribución (artículo, noticias breves/nota informativa, recensión, debate); número de páginas; idioma.

No siempre ha resultado fácil hacer la distinción entre artículos cortos y noticias breves/notas informativas. Se ha tenido fundamentalmente en cuenta para ello la orientación del trabajo (más reflexivo que informativo) y, en menor medida, su extensión.

b) Orientación: temática principal (estudios de crono-tipología, arqueología del paisaje, arqueometría, arte, datación absoluta, trabajos de síntesis/revisión, estudios de paleoambiente, otros); método de intervención (excavación, prospección, análisis de la cultura material, análisis espacial y estadístico, análisis paleobiológico y sedimentológico); período estudiado (Neolítico, Calcolítico, Campaniforme, Edad del Bronce, Edad del Hierro, diacrónico, indeterminado); tipo de monumentos (túmulo/cista, menhir/círculo lítico, bloque natural, otros); aproximación teórica.

En la temática principal 'otros' hace referencia a cuestiones numéricamente poco representadas en la muestra (e.g. trabajos de conservación, listados de inventarios, cálculos de demografía en la Prehistoria, publicación de correspondencia de autores clásicos); el valor de algunas de estas temáticas se analizará sin embargo en el apartado de discusión. La datación absoluta se ha individualizado del marco general de los estudios de Arqueometría. A pesar de que el Neolítico ocupa un lugar central en la consideración de la monumentalidad prehistórica el fenómeno sobrepasa por sus características los límites cronológicos establecidos para ese período; la referencia a múltiples períodos aparece claramente reflejada en la muestra y se hace necesaria. En el análisis de los tipos arquitectónicos la variabilidad de denominaciones, el estado de conservación de los monumentos y la indefinición de algunos autores a la hora de especificar las estructuras estudiadas nos ha llevado a agrupar bajo una misma denominación los yacimientos de carácter tumular, ortostático y cistas; se ha incluido una tipología referente a afloramientos naturales, así como una mención a 'otros' a causa de unos pocos trabajos que analizan contextos habitacionales integrando en la discusión la monumentalidad. La aproximación teórica de los trabajos será considerada de modo muy general; pocos trabajos hacen explícitas las bases de sus aproximaciones y su clasificación no siempre resulta evidente a partir de su lectura.

c) Autoría: número de autores; institución; nacionalidad; género.

Se ha tenido en cuenta para el cómputo de la nacionalidad el lugar al que pertenece la insti- 


\begin{tabular}{|c|c|c|c|}
\hline Publicación & Contidad & Tipo & Ambito \\
\hline ANTRQUITY & 1 & REVISTA & INTERNACIONAL \\
\hline ANUARIO BRIGANTINO & 1 & REVISTA & REGIONAL \\
\hline ARKEOS & 1 & REVISTA & NACIONAL \\
\hline ARQUEOLLOGIA (Porto) & 1 & REVISTA & NACIONAL \\
\hline ARQUIVOS DO SEMINARIO DE ESTUDOS GALEGOS & 1 & REVISTA & REGIONAL \\
\hline BOLETIN AURIENSE & 1 & REVISTA & REGIONAL \\
\hline BOLETIN DE LA COMIS. PROV. DE MONUM. HISTORICOS Y ARTISTICOS DE LUGO & 1 & REVISTA & REGIONAL \\
\hline BOLETIN DE LA COMIS. PROV. DE MONUM. HISTORICOS Y ARTISTICOS DE ORENSE & 1 & REVISTA & REGIONAL \\
\hline BOLETIN DE LA REAL ACADEMIA GAUEGA & 1 & REVISTA & REGIONAL \\
\hline BOLETIN DEL SEMINARIO DE ESTUDIOS DE ARTE Y ARQUEOLOGIA & 1 & REVISTA & REGIONAL \\
\hline BOLETIN DO MUSEO PROVINCAL DE LUGO & 1 & REVISTA & REGIONAL \\
\hline BRIGANTIUM & 1 & REVISTA & REGIONAL \\
\hline CADERNOS DE ARQUEOLOGIA E PATRIMONIO & 1 & REVISTA & REGIONAL \\
\hline CADERNOS DE ARQUEOLOGIA, Serie II & 1 & REVISTA & REGIONAL \\
\hline CASTRELOS: Revista do Museo Municipal "Quñones de León" & 1 & REVISTA & REGIONAL \\
\hline COLOQUIO DE ARQUEOLOGIA DO NOROESTE PENINSULAR & 1 & CONGRESO & PENINSULAR \\
\hline COLOQUIO DE MEGALITSMO DE REGUENGOS DE MONSARAZ (I \& II) & 2 & CONGRESO & INTERNACIONAL \\
\hline COMPLUTUM & 1 & REVISTA & NACIONAL \\
\hline CONGRESO DE ARQUEOLOGIA PENINSULAR (I, II, III) & 3 & CONGRESO & PENINSULAR \\
\hline CONGRESO DE NEOUTICO PENINSULAR $(\mathrm{l}, \mathrm{Il}, \mathrm{III})$ & 3 & CONGRESO & PENINSULLAR \\
\hline CONGRESO NACIONAL DE ARQUEOLOGIA (España) (III, XIV, XVIII, XXII) & 4 & CONGRESO & NACIONAL \\
\hline CONGRESSO NACIONAL DE ARQUEOLOGIA. (Portugal) & 1 & CONGRESO & NACIONAL \\
\hline CONGRESSO TRANSFRONTEIRIÇO DE ARQUEOLOGIA - MONTALEGRE & 1 & CONGRESO & REGIONAL \\
\hline CONIMBRIGA & 1 & REVISTA & REGIONAL \\
\hline CUADERNOS DE ESTUDIOS GALLEGOS & 1 & REVISTA & REGIONAL \\
\hline EL MUSEO DE PONTEVEDRA & 1 & REVISTA & REGIONAL \\
\hline ERA ARQUEOLOGIA & 1 & REVISTA & NACIONAL \\
\hline ESPACIO, TEMPOY FORMA & 1 & REVISTA & REGIONAL \\
\hline ESTUDOS PRE-HISTORICOS & 1 & REVISTA & NACIONAL \\
\hline EUROPEAN JOURNAL OF ARCHAEOLOGY & 1 & REVISTA & INTERNACIONAL \\
\hline GALLAECIA & 1 & REVISTA & REGIONAL \\
\hline O NEOUTICO ATLANTICO E AS ORIXES DO MEGALITISMO & 1 & CONGRESO & INTERNACIONAL \\
\hline JOURNAL OF IBERIAN ARCHAEOLOGY & 1 & REVISTA & PENINSULLAR \\
\hline L'ANTHROPOLOGIE & 1 & REVISTA & INTERNACIONAL \\
\hline LE MEGAUTHISME ATLANTIQUE: ACTES DU XIVEME CONGRES UISPP & 1 & CONGRESO & INTERNACIONAL \\
\hline MADRIDER MITTEILUNGEN & 1 & REVISTA & PENINSULAR \\
\hline MESA REDONDA SOBRE MEGALUTISMO PENINSULAR-AEAA & 1 & CONGRESO & PENINSULAR \\
\hline MINIUS & 1 & REVISTA & REGIONAL \\
\hline O ARQUEOLOGO PORTUGUES (Series I, II, IV) & 3 & REVISTA & NACIONAL \\
\hline OXFORD JOURNAL OF ARCHAEOLOGY & 1 & REVISTA & INTERNACIONAL \\
\hline PONTEVEDRA ARQUEOLOGICA & 1 & REVISTA & REGIONAL \\
\hline PORTUGALla (Série Antiga, Nova Série) & 2 & REVISTA & NACIONAL \\
\hline REVISTA DA FACULDADE DE LETRAS DO PORTO - HISTORIA & 1 & REVISTA & REGIONAL \\
\hline REVISTA DE CIENCLAS HISTORICAS (Universidade Portucalense) & 1 & REVISTA & REGIONAL \\
\hline REVISTA DE GUMMARAES & 1 & REVISTA & REGIONAL \\
\hline REVISTA PORTUGUESA DE ARQUEOLOGIA & 1 & REVISTA & NACIONAL \\
\hline SAGUNTUM & 1 & REVISTA & NACIONAL \\
\hline SEMINARIO DE ARQUEOLOGIA DO NOROESTE PENINSULAR (I \& II) & 2 & CONGRESO & PENINSULAR \\
\hline SETUBAL ARQUEOLOGICA & 1 & REVISTA & NACIONAL \\
\hline SPAL & 1 & REVISTA & NACIONAL \\
\hline TRABANOS DE PREHISTORLA & 1 & REVISTA & NACIONAL \\
\hline TRABALHOS DA SOCIEDADE PORTUGUESA DE ANTROPOLOGIA E ETNOLOGIA & 1 & REVISTA & NACIONAL \\
\hline TRABALHOS DE ANTROPOLOGIA E ETNOLOGIA & 1 & REVISTA & NACIONAL \\
\hline ZEPHYRUS & 1 & REVISTA & NACIONAL \\
\hline Subtotal revistos & 46 & & \\
\hline Subtotal congresos & 20 & & \\
\hline Total & 66 & & \\
\hline
\end{tabular}

Tabla 1.- Listado de publicaciones que contenían información para el análisis 
tución, y no la nacionalidad de los autores propiamente dicha. Se han registrado varios centros por autor en el caso de investigadores que han trabajado en varias instituciones a lo largo de su vida profesional. Se ha registrado el género de los autores; en los trabajos firmados por un colectivo se ha considerado una categoría de género 'mixto'.

d) Ámbito geográfico estudiado: término municipal; provincia o distrito; país.

Del total de 83 publicaciones del listado inicial, 66 contenían información relativa al objeto de estudio (Tabla 1). De éstas 46 corresponden a revistas y 20 a congresos. El vaciado ha dado finalmente lugar a un total de 632 registros y 283 autores. El tamaño de la muestra en otros estudios bibliométricos similares ha sido de 1440 (Montero Ruiz et al. 2007), 521 (Cruz Berrocal et al. 1999), 245 (López-Romero y Montero Ruiz 2006), 223 (García-Heras 1997), 201 (Palomar et al. 2009), 59 (Cordero et al. 2006). Los estudios de M. García-Heras y de T. Cordero et al. se extendían no obstante al número de muestras analizadas en cada artículo (6716 y 1382 respectivamente).

El rango cronológico de la muestra comprende desde 1888 hasta 2010. Como tal muestra este conjunto de datos no refleja la totalidad de la producción científica aunque constituye, por el rango cronológico y número de fuentes revisadas, un porcentaje de ésta que consideramos representativo.

\section{Análisis}

Teniendo presentes los bloques de variables la exposición de los análisis realizados se dividirá a su vez en cuatro apartados: análisis formal; análisis temático; autoría; análisis regional.

\subsection{Análisis formal}

El 36.8\% de las 66 publicaciones analizadas son revistas o congresos de carácter regional (centradas en Galicia o en el Norte de Portugal), el 34.8\% revistas o congresos de carácter nacional (centradas en Portugal o en España), el 21.2\% son de ámbito peninsular (Portugal y España) y el 9.1\% son internacionales. A pesar de su papel regional la revista Arqueologia (Porto) publica trabajos de diversas regiones de Portugal, por lo que ha sido finalmente considerada como nacional. Por su limitado alcance geográfico (Trás-os-Montes Occidental y Ourense) el congreso transfronterizo de Montalegre (3-5 de octubre de 2008) ha sido considerado como regional. Las Comunicações dos Serviços Geológicos

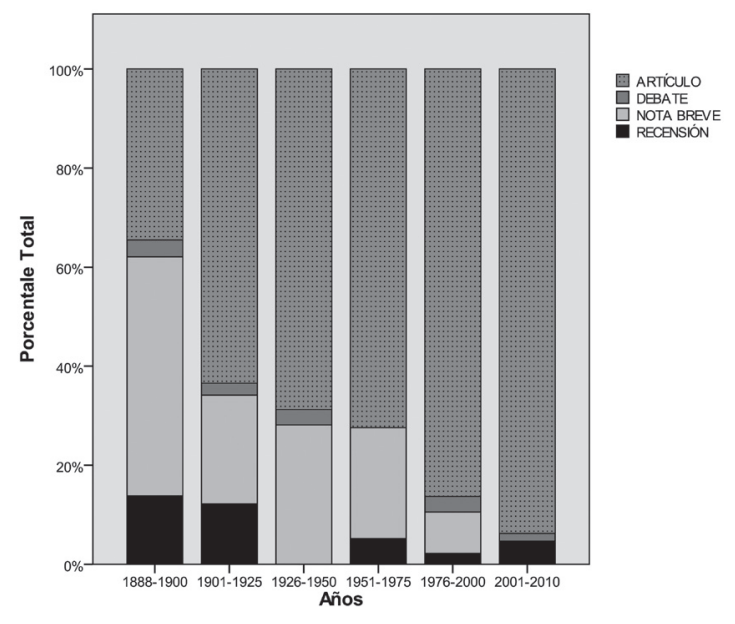

Fig 2.- Tipos de trabajos por bloques de 25 años.

de Portugal no han sido finalmente vaciadas por la dificultad que tuvimos para acceder a las series completas.

Al analizar el número de trabajos publicados se hace evidente el predominio de los ámbitos regional (279 trabajos en revistas o congresos regionales, el $44.1 \%$ del total) y nacional (259 trabajos, 41\%), frente a la menor incidencia de trabajos en series peninsulares (60 trabajos, 9.5\%) e internacionales (34 trabajos, 5.4\%).

Dentro de la muestra se observan distintos momentos de intensidad en las publicaciones. Un primer momento destacado se produce en los años finales del siglo XIX y principios del siglo XX (1895-1904), cuando documentamos un total de 45 trabajos. Entre

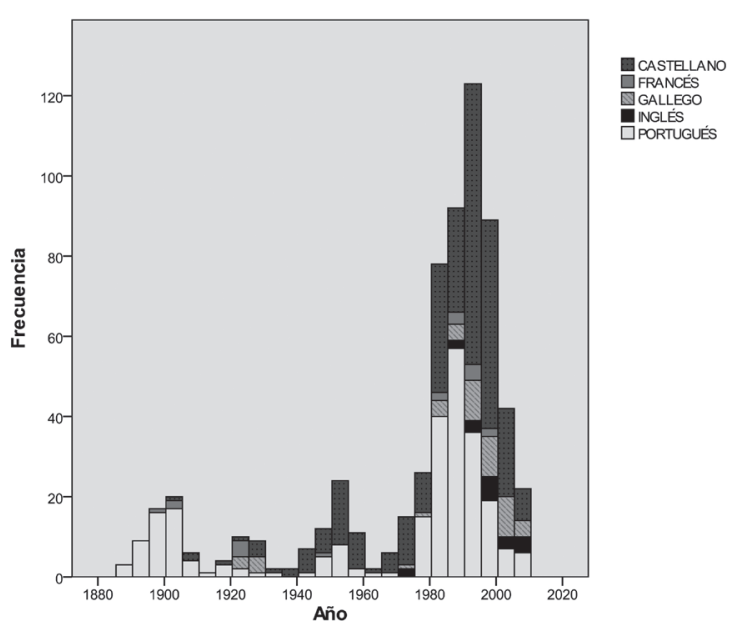

Fig 3.- Distribución cronológica y frecuencia de uso de los idiomas. 


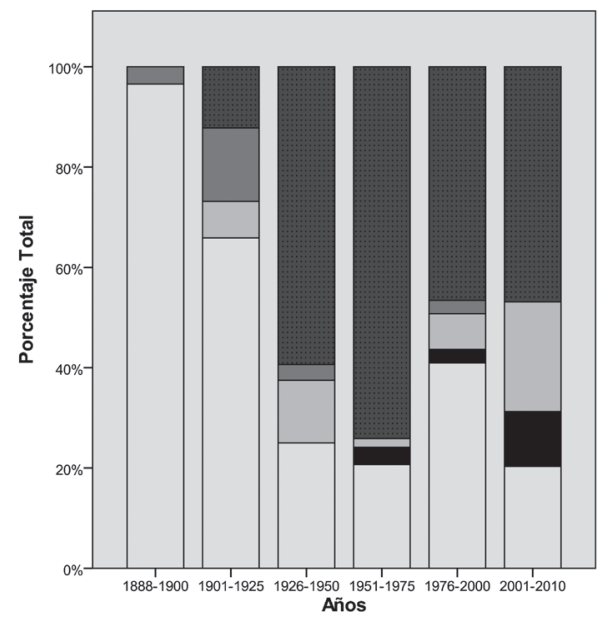

Fig. 4.- Idiomas por bloques de 25 años.

1947 y 1956 la muestra recoge un total de 42; destaca en este momento la presencia de diez trabajos en 1955, siete de los cuales corresponden a la publicación de las ponencias del tercer Congreso Nacional de Arqueología (Santiago de Compostela, 1953). El gran salto en el estudio de la monumentalidad en la región se produce entre 1980 y 2003; a lo largo de este período se publican más de seis trabajos al año, con la excepción de 1996, y se alcanzan máximos de 29 (1988 y 2000), 32 (1994 y 1997) y 40 (1995). Es también el momento en el que nacen o se consolidan revistas vinculadas a los centros de investigación más activos en la región. Tras este período el número de publicaciones decrece progresivamente, sólo superándose en 2005 y 2006 los cinco trabajos anuales.

Respecto al tipo de trabajos predominan los artículos (512 trabajos, el 81\% del total), seguidos por noticias breves/notas informativas (79 trabajos, $12.5 \%)$.

Las recensiones son poco frecuentes (24 trabajos, 3.8\%), al igual que lo es la publicación de debates (17 debates publicados, el $2.7 \%$ del total de trabajos). A pesar de ser una segmentación un tanto artificial, los porcentajes del tipo trabajos por períodos de 25 años dan una mejor idea de la evolución del modo de publicación (fig. 2). Teniendo presente el sesgo producido en los extremos de dicha segmentación (inicio en 1888 en lugar de 1875 y fin en 2010 en lugar de 2025) la tendencia más marcada es el incremento del número de artículos frente al resto de formatos. Los debates se mantienen en porcentajes bajos en toda la serie, desapareciendo durante el período 1951-1975; las noticias breves/notas informativas son más frecuentes en el período inicial, si bien se mantienen en porcentajes superiores al $20 \%$

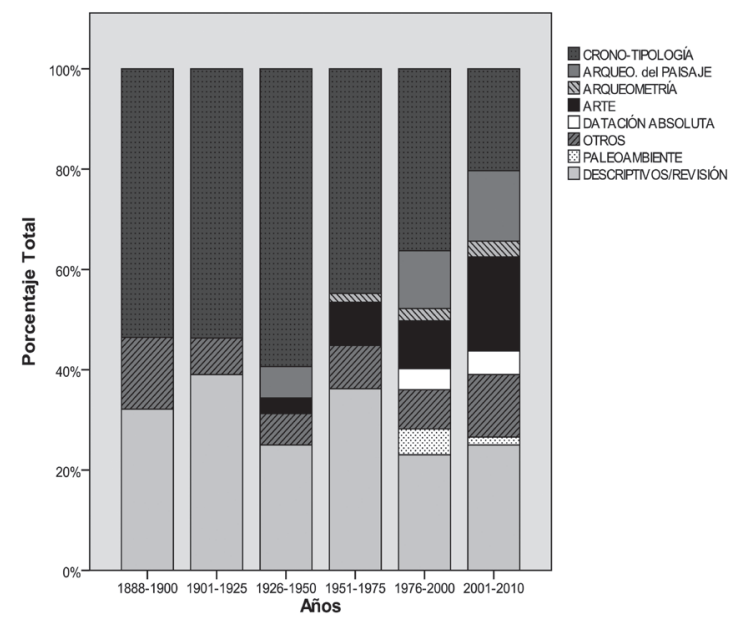

Fig. 5.- Temáticas por bloques de 25 años.

del total hasta el último tercio del siglo XX; el porcentaje de recensiones es variable, siendo superior al $15 \%$ del total de trabajos hasta el primer cuarto del siglo XX, desapareciendo durante el segundo cuarto del siglo y manteniéndose en porcentajes inferiores al 10\% entre 1951 y 2010.

La extensión media de los artículos es de 18.36 páginas, con un máximo de 181 que corresponde a un trabajo de 1956 sobre Pontevedra a cargo de J. Filgueira Valverde. Otros dieciocho trabajos superan las 50 páginas de extensión; se trata principalmente de artículos de síntesis, revisión teórico-metodológica o catalogación. El valor modal (número de páginas/artículo más repetido) es de diez páginas y se repite en 32 artículos.

La extensión media de las noticias breves/notas informativas es de 3.18 páginas, con un valor modal de 2 páginas que se repite en un total de 24 casos. Sólo 7 de las 79 noticias superan las 5 páginas de extensión. Los debates tienen una extensión media de 9.80 páginas (valor modal de 5 , con dos únicos casos), mientras que las recensiones no superan las 5 páginas de media (4.48 páginas; valor modal de 2 páginas que se da en 9 casos).

Los idiomas de publicación registrados son el inglés, francés, gallego, portugués y castellano. El idioma que predomina es el castellano (287 trabajos, el $45.4 \%$ del total), seguido del portugués (255, $40.3 \%$ ) y del gallego $(51,8.1 \%)$, mientras que el inglés $(20,3.2 \%)$ y el francés $(19,3 \%)$ son minoritarios. La distribución cronológica del uso de los distintos idiomas se representa en la figura. 3. Además del predominio del portugués en los primeros años recogidos en la muestra, destaca la desaparición del gallego entre 1930 y mediados de la déca- 
da de 1970. El predominio del castellano sobre el portugués se hace evidente de 1950 a $1970 \mathrm{y}$, sobre todo, en la década de 1990. Mientras que el francés aparece de forma intermitente a lo largo de toda la secuencia (e.g. 1896, 1904, 1923, 1947, 1983...), el inglés se incorpora sólo en época reciente (1973), (fig. 4).

\subsection{Análisis temático}

Se registró la orientación principal de cada uno de los 632 trabajos que componen la muestra, identificándose ocho bloques. Los estudios de crono-tipología predominan claramente (244 trabajos, 38.6\% del total), seguidos de los trabajos de síntesis/revisión $(164,25.9 \%$ del total). La representatividad del resto de bloques no supera el $10 \%$ del total de trabajos. Destacan no obstante 58 trabajos sobre arqueología del paisaje $(9.2 \%$ del total) y $57(9 \%)$ sobre 'arte megalítico'. Un total de 22 trabajos (3.5\%) tratan aspectos paleoambientales relacionados con los monumentos, $20(3.2 \%)$ se centran en la datación absoluta, mientras que 13 (2.1\%) corresponden a análisis arqueométricos. Finalmente, el 8.5\% (54 trabajos) incluye otras aproximaciones con escasa representatividad (estudios sobre demografía, publicación de correspondencia, etc.).

A pesar de su predominio los trabajos de cronotipología han ido perdiendo representatividad. Este declive se hace patente a partir de 1995. Por otro lado, es interesante constatar que los trabajos de síntesis se dan en la región desde el inicio, manteniendo en la distribución por series de 25 años porcentajes por encima del $20 \%$ del total de publicaciones (fig. 5). Si bien desde el punto de vista de la historia de la disciplina la Arqueología del Paisaje se desarrolla en el último tercio del siglo XX, la perspectiva llevada a cabo por F. Maciñeira (1870-1943) en la región de Ortigueira (A Coruña) justifica la presencia de dos trabajos de este tipo en momentos tempranos (1929 y 1930). Los trabajos sobre paleoambiente aparecen asociados al momento de máxima expansión de las publicaciones; el primer trabajo de este estilo registrado en la muestra es un pequeño artículo de 1982 en la revista Arqueologia que analiza los restos vegetales recogidos en monumentos del norte de Portugal entre 1978 y 1981. Algo similar ocurre con la introducción del radiocarbono como método de datación absoluta; el primer trabajo de este tipo registrado para el norte de Portugal data de 1983 (túmulo de Meninas de Castro, Porto), mientras que para Galicia las dos primeras dataciones son publicadas en 1985 (túmulos de As Rozas I y Chan da Cruz, región de Pontevedra). El análisis arqueométrico de materias primas asociadas a los monumentos es relativamente temprano; un primer

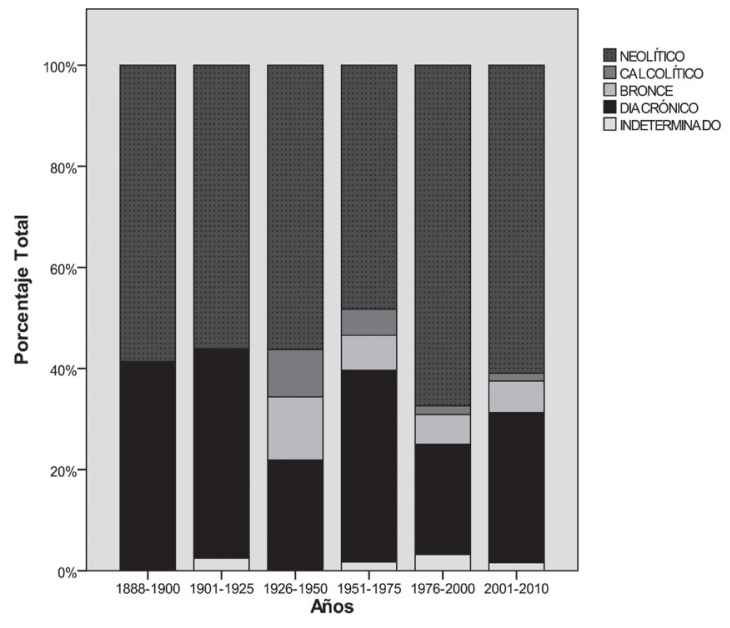

Fig. 6.- Período estudiado por bloques de 25 años.

estudio de composición de dos cuentas de variscita procedentes del túmulo 7 de Monte da Morá (región de Lugo) es publicado en 1975. Los estudios con temática principal sobre arte megalítico se inician en la muestra con un trabajo de F. Bouza-Brey (1943), si bien se consolidan únicamente a partir de la década de 1970. Existe no obstante una referencia a grabados realizada por el mismo autor en 1927 que por su orientación principal ha sido clasificado dentro del bloque de estudios de crono-tipología.

Un segundo aspecto del análisis temático tiene que ver con los modos de intervención empleados en las distintas investigaciones publicadas. Dejando al margen los métodos propios a los trabajos de revisión (crítica bibliográfica y crítica teórica) así como algunos métodos específicos de catalogación (e.g. calcos de grabados y pinturas) y puesta en valor, nos centraremos en las características más específicamente analíticas del conjunto de datos: excavación, prospección, análisis de la cultura material, análisis espacial y estadístico, análisis paleobiológico y sedimentológico. El conjunto 'análisis de cultura material' engloba en este caso tanto a los estudios tipológicos como a los arqueométricos.

La simplificación de la muestra en estos cinco tipos da como resultado un subtotal de 430 trabajos. Destacan los trabajos de excavación $(145,33.7 \%$ del total de 430), seguidos por los estudios de cultura material (112 trabajos, 26\%). El análisis espacial y estadístico se da en 83 trabajos (19.3\%), mientras que la prospección arqueológica supone el 14.7\% (63 trabajos). El menor porcentaje viene dado por los análisis paleobiológicos y sedimentológicos $6.3 \%$ (27 trabajos), que se incorporan de forma algo más tardía que el resto. 


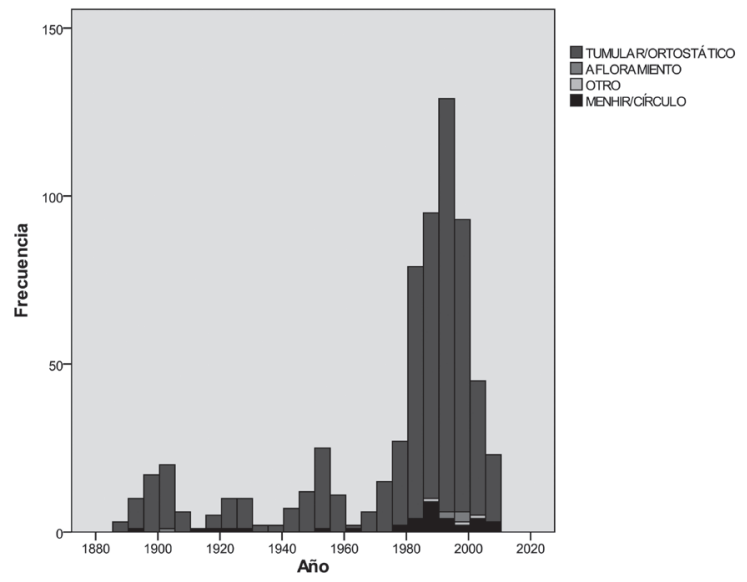

Fig. 7.- Distribución cronológica y frecuencia de los tipos de monumentos estudiados.

Si nos referimos únicamente al conjunto de intervenciones de campo (208 trabajos sumando excavaciones y prospecciones) el $69.7 \%$ son trabajos de excavación mientras que las prospecciones ocupan el $30.3 \%$ restante. Sólo durante el segundo y tercer cuarto del siglo XX las prospecciones superan porcentualmente a los trabajos de excavación. No se documenta en la muestra la publicación de ninguna intervención de campo entre los años 1931 a 1943 y 1957 a 1967 . La inmensa mayoría de estos trabajos de campo publicados son intervenciones programadas $(90.9 \%)$, frente al $9.1 \%$ de intervenciones de urgencia. Estas últimas se concentran entre los años 1982 y 2003; como única y curiosa excepción a esta dinámica cabe señalar un trabajo de 1895 en O Arqueôlogo Portugués y que constituye uno de los primeros testimonios de excavación destinada a evitar la expoliación de los monumentos (Rodrigues 1895). El artículo de X.I. Vilaseco Vázquez (2001) sobre las metodologías de excavación del megalitismo gallego entre 1965 y 1998 constituye un valioso complemento para la comprensión de estas dinámicas.

Un tercer aspecto del análisis temático se centró en el período cronológico estudiado. Como ha quedado dicho la discusión sobre la monumentalidad excede los límites del Neolítico. Así, se han podido definir cuatro contextos cronológicos: Neolítico; Calcolítico; Edad del Bronce; diacrónico. A ellos hay que sumar un grupo de trabajos de cronología indefinida.

Más de la mitad de los trabajos (400, el 63.3\% del total de 632) tratan contextos Neolíticos; sólo 44 de ellos introducen de forma explícita algún tipo de subdivisión, que generalmente suele li- mitarse a las denominaciones de Medio y Final. Un segundo grupo destacado es el de los trabajos que analizan dos o más períodos; este bloque (166 trabajos, $26.3 \%$ del total) está fundamentalmente compuesto por estudios que discuten conjuntamente el Neolítico y la Edad del Bronce, y por algunos trabajos de prospección cuyo espectro cronológico puede llegar a cubrir desde el Paleolítico hasta la Edad Media. Los estudios sobre Calcolítico son los que menor representación tienen (14 trabajos, $2.2 \%$ del total), mientras que 36 trabajos (5.7\% del total) analizan la monumentalidad en la Edad del Bronce; un porcentaje importante de éstos $(15$, el $41.6 \%$ del total de 36$)$ se centra en la presencia de cerámica campaniforme en contextos tumulares. Por último en 16 trabajos $(2.5 \%$ del total de 632) no se ha podido identificar con claridad el período estudiado.

Hasta el segundo cuarto del siglo XX las menciones a la monumentalidad hacen referencia a contextos neolíticos o se enmarcan en trabajos de contenido diacrónico. Los primeros trabajos de la muestra que discuten explícitamente la presencia de elementos calcolíticos o posteriores en contextos tumulares son dos artículos de 1927 (Pericot García 1927; López Cuevillas 1927). Los porcentajes de representatividad de estos períodos se mantienen en niveles bajos a lo largo de toda la secuencia (fig. 6).

El análisis tipológico ha ocupado un lugar destacado. Puesto que varios trabajos analizan más de un tipo de monumentos (23 registros, el 3.5\%, hacen referencia dos o más tipologías) el número de registros para esta sección es de 655 en lugar de los 632 habituales.

El 93.3\% de los yacimientos estudiados (611 de los 655) son de tipo tumular. Sólo el 5.3\% (35 registros) se centran en el análisis de menhires o círculos líticos. Las discusiones sobre éstos son marginales a lo largo de toda la muestra, y sólo en la década de 1980 se observa una cierta expansión de las mismas (12 trabajos); entre 2001 y 2010 su representatividad alcanza el $10 \%$, algo que se explica en parte por la fuerte caída del número general de trabajos sobre el fenómeno tumular. La discusión del carácter monumental de determinados afloramientos naturales y de su papel de estructuración del paisaje para las sociedades constructoras de monumentos resulta mucho más marginal, siendo objeto de análisis en 6 ocasiones $(0.9 \%)$. La primera de ellas presente en la muestra data de 1901 (Vasconcellos 1901) y ninguna otra reflexión al respecto vuelve a aparecer hasta 1993 (Criado Boado 1993). Tres registros (0.5\% del total de 655) discuten conjuntamente los contextos habitacionales y funerarios en momentos neolíticos y de la Edad del Bronce (fig. 7). 


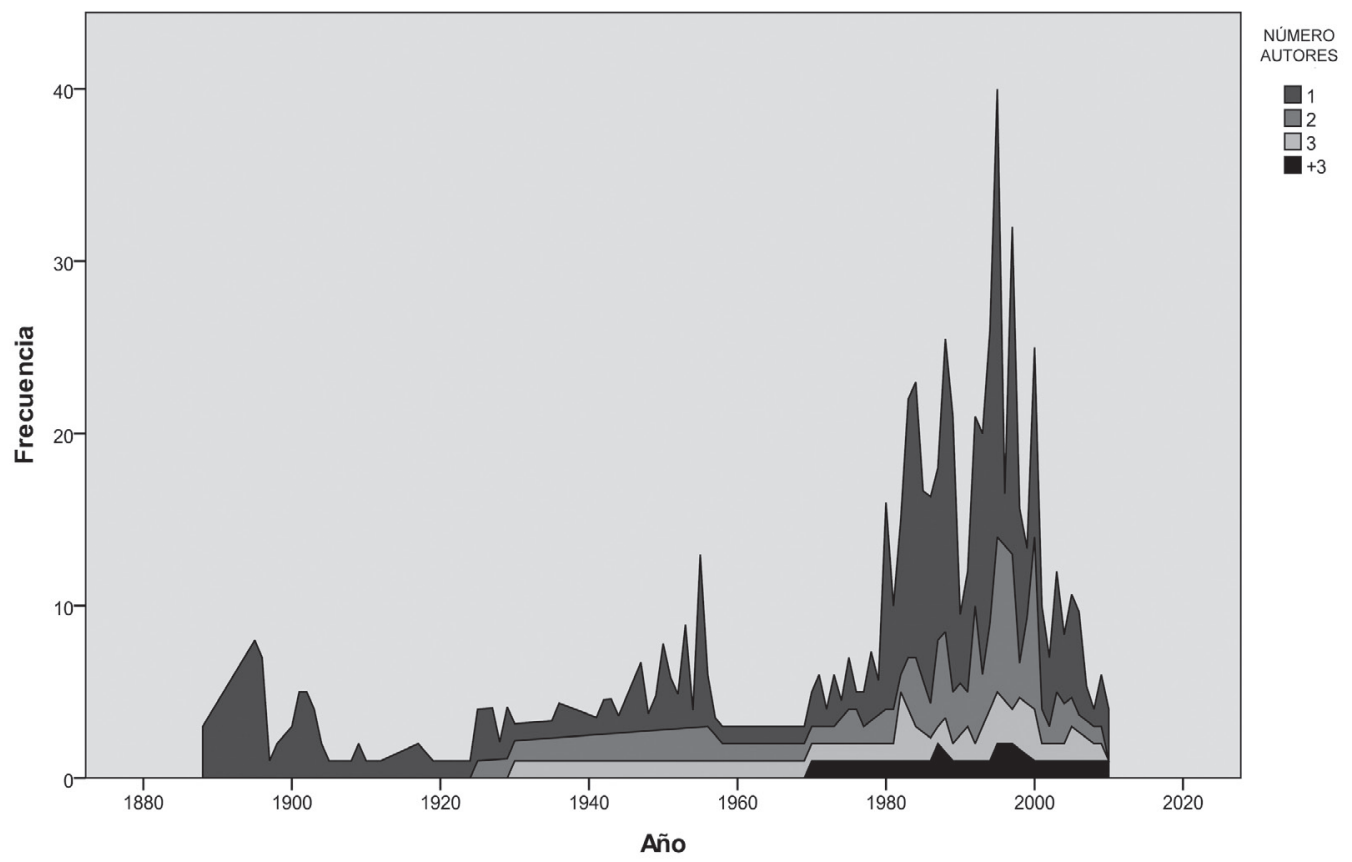

Fig. 8.- Distribución cronológica y frecuencia del número de autores.

El estudio de la aproximación teórica de los trabajos es complejo (falta de toma de postura explícita de los autores, importancia cuantitativa de las síntesis y revisiones, presencia de recensiones y notas breves, etc.). Pese a ello hemos realizado una caracterización sucinta de los planteamientos de investigación. La mayoría de contribuciones son de corte histórico-cultural (222 trabajos, 35.1\% del total). Las de tipo procesual son igualmente muy numerosas, situándose en valores próximos a las anteriores (214 trabajos, 33.9\%). Un total de 82 trabajos (13\% del total) son de corte postprocesual. Entre ellos, tres artículos publicados en 1998, 2000 y 2009 presentan una orientación que puede ser considerada fenomenológica. Destaca la ausencia de trabajos adscribibles al conjunto de arqueologías marxistas; sólo Bello Diéguez et al. (1982) presentan una discusión sobre el contexto social del megalitismo gallego con términos afines a esta tendencia. El porcentaje restante $(17.4 \%$, 110 trabajos) no ha podido ser contextualizado con claridad por los motivos ya señalados.

Si tomamos únicamente el período a partir de 1975, momentos de desarrollo y consolidación de los postulados procesuales y postprocesuales, la representatividad varía sensiblemente. Dominan las perspectivas procesuales (205 trabajos, $43.4 \%$ ), seguidas de las histórico-culturales (102, $21.6 \%)$ y postprocesuales $(82,17.6 \%)$.

\subsection{Autoría}

Se han registrado 283 autores, que pueden haber firmado uno o más trabajos en solitario o en colaboración.

Atendiendo al número de trabajos por autor, sólo 16 de los 283 (el 5.6\%) firman en más de 10 trabajos, lo que es indicativo de una cierta diversificación de la producción. El autor con más trabajos en la muestra es V.O. Jorge (64 firmas), seguido de R. Fábregas Valcárce (47), F. Criado Boado (34), J.M. Vázquez Varela (30), M.J. Sanches (19) y F. López Cuevillas (18). El Grupo de Estudos Arqueológicos do Porto, firma colectiva que engloba a algunos de los 16 autores destacados, aparece en 11 trabajos.

La producción por años pone de manifiesto el peso de los autores portugueses en la investigación de finales del siglo XIX y del primer cuarto del siglo XX. J. Leite de Vasconcellos (17 trabajos entre 1895 y 1922), F. Martins Sarmento (7 trabajos entre 1888 y 1902, más uno póstumo en 1970) y H.M.F. Botelho (7 trabajos entre 1898 y 1905) concentran la producción en esos momentos. A partir del segundo cuarto del siglo XX los autores españoles comienzan a destacar. F. López Cuevillas es uno de los que más continuidad presenta; su primer trabajo en la muestra data de 1925 y el último de 1960, siendo su producción especialmente activa entre finales de la década de 1940 y finales de la de 1950. A partir de 


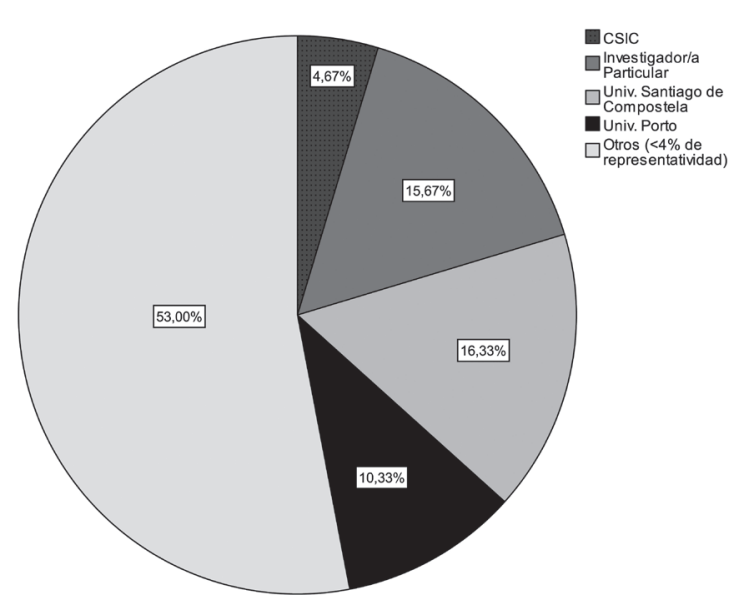

Fig. 9.- Centros de procedencia de los autores.

la segunda mitad del siglo XX el número de autores aumenta de forma significativa, algo que se hace patente en el incremento de publicaciones.

Para el análisis de la coautoría se han eliminado de la muestra las recensiones y los debates. Ambos introducen un sesgo: las recensiones por estar realizadas por un único autor (sólo una reseña publicada en Gallaecia en 2000 tiene dos firmantes), los debates por agrupar a múltiples autores en torno a un tema de discusión sin que pueda considerarse un trabajo colectivo. Esto deja un conjunto de 591 trabajos del total de 632. El 72.9\% (431) de ellos ha sido realizado por un solo autor. El 18.1\% (107) resulta de la colaboración de dos autores, mientras que sólo el 6.8\% (40) corresponde a trabajos firmados por tres autores. En 13 trabajos (2.3\%) firman cuatro o más autores, siendo nueve el máximo número de autores registrado. Así pues, el porcentaje de trabajos con más de un autor es del $27.1 \%$. Este porcentaje de coautoría es ligeramente inferior a algunos publicados en otros estudios bibliométricos (e.g. 28.52\% en Rodríguez Alcalde et al. 1993: 22; 32.3\% en Rodríguez Alcalde et al. 1996: 45).

La evolución de la coautoría por años se muestra en la figura 8. El primer artículo de la muestra con más de tres autores data de 1970. A partir del año 2000 se observa un ligero descenso en el porcentaje de trabajos firmados por dos autores en favor de los trabajos individuales y de los trabajos firmados por tres autores.

En lo que respecta a la procedencia geográfica se han establecido dos niveles de análisis. Por un lado, se han analizado las localidades en las que se ubican los centros de investigación a los que cada autor se adscribe; por otro lado, se han considerado los países que acogen a dichas instituciones. Puesto

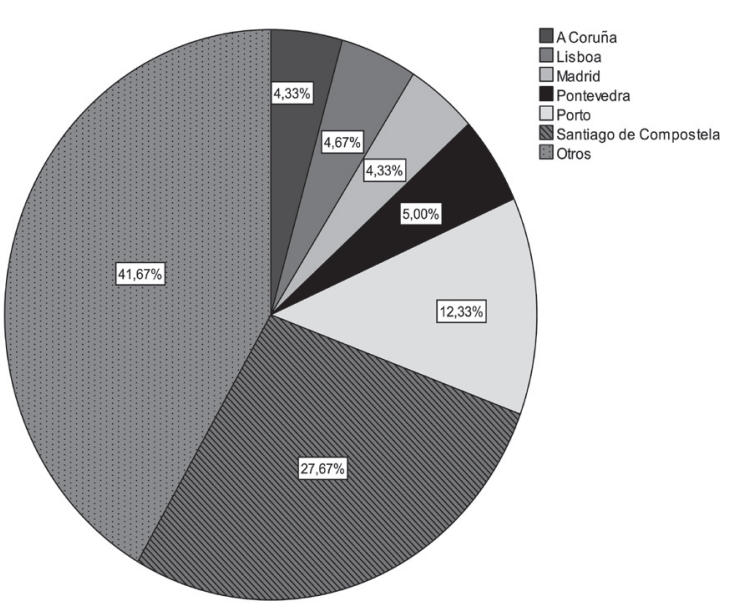

Fig. 10.- Localidades de procedencia de los autores.

que se observó que algunos autores pertenecieron a lo largo de su carrera a más de una institución, un análisis previo ha de consistir en la caracterización de esta movilidad profesional.

La gran mayoría de autores (268, el 94.73\% del total de 283) firman como miembros de una sola institución. 14 (4.92\%) firman desde posiciones en dos instituciones diferentes, mientras que sólo uno $(0.35 \%)$ ha pertenecido a tres organismos. De estos 15 que han pertenecido a más de una institución sólo tres son autores activos durante la primera mitad del siglo XX; aunque esta observación ha de ser tomada con cautela por la naturaleza de la muestra es posible ver en esta tendencia un incremento de la movilidad profesional, algo que se hace especialmente patente en las últimas décadas.

La institución que más investigadores aporta es la Universidade de Santiago (48 investigadores), seguida por la Universidade do Porto (31) y por el CSIC (14). Un total de 47 autores han sido clasificados como 'investigadores particulares' al no haber constancia de su pertenencia a ninguna institución oficial en el momento de firmar sus trabajos (fig. 9). Del mismo modo, no ha sido posible establecer el origen de diez autores españoles y seis portugueses, habiendo sido clasificados como 'investigadores de procedencia no definida'.

Teniendo en cuenta que algunos de los 283 autores han trabajado en más de una institución, y que éstas se encuentran en ocasiones en ubicaciones diferentes, contamos con un total de 299 registros para el análisis de las localidades (fig. 10). Santiago de Compostela es la localidad que ha aportado mayor número de investigadores (83 registros en el cómputo de los 299 autores/institución); además de la Universidade de Santiago en la localidad se ubican o ubicaron entre 


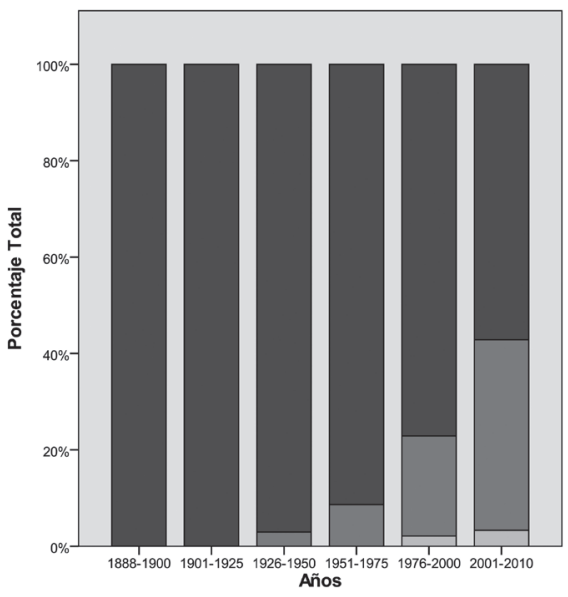

Fig. 11.- Género.

otros el Seminario de Estudos Galegos, el Instituto de Estudos Galegos Padre Sarmiento y varios centros de la Xunta de Galicia. Le siguen la ciudad de Porto con 37 (investigadores de la Universidade do Porto y Universidade Portucalense), la ciudad de Pontevedra con 15 (Grupo de Arqueología-Alfredo García Alén, Museo de Pontevedra, Escola Superior de Conservación e Restauración de Bens Culturais, etc.) y la ciudad de Lisboa con 14 (Universidade de Lisboa, Serviços Geológicos de Portugal, Academia Portuguesa da Historia, Museu Nacional de Arqueologia, etc.). Siguen ciudades como A Coruña y Madrid (13),
Lugo y Ourense (11), Barcelona (9), Guimarães (6), Coimbra, Sacavem y Paris (5).

Estas localidades se encuentran ubicadas en un total de 6 países. La mayoría de los autores/institución se ubican en España (180, el $60.2 \%$ del total de 299), mientras que los ubicados en Portugal son casi exactamente la mitad $(91,30.4 \%$ del total). Los otros países son Francia (16 registros, 5.4\%), Reino Unido (9 registros, 3\%), EEUU (2 registros, $0.7 \%$ ) y Méjico ( 1 registro, $0.3 \%$ ). La presencia de este último se debe a la publicación de un artículo de P. Bosch-Gimpera en la Revista de Guimarães durante su etapa como profesor en la Universidad Nacional Autónoma de México.

Al margen de esta distribución nos interesa saber en qué medida la investigación regional se ha apoyado en la colaboración internacional y transfronteriza a la hora de abordar el objeto de estudio. Sólo 12 trabajos están firmados por dos o más autores procedentes de distintos países. Si descartamos los registros correspondientes a debates y recensiones (por las razones ya esgrimidas), estos 12 trabajos de coautoría internacional representan únicamente el $2 \%$ de la producción. Las colaboraciones implican a investigadores de España y Reino Unido (4 trabajos), de Portugal y Francia (3), de Portugal y EEUU (1) y de España y Portugal (4). Estos trabajos de colaboración hispanoportuguesa representan únicamente el $0.67 \%$; la gran mayoría de estas coautorías se fundamentan además en la caracterización arqueométrica de materiales y en la datación de yacimientos portugueses por laboratorios españoles externos al noroeste, no siendo pro-

Tabla de contingencia Temática * Género

\begin{tabular}{|c|c|c|c|c|c|}
\hline & & & \multicolumn{2}{|c|}{ genero } & \multirow[b]{2}{*}{ Total } \\
\hline & & & $\mathrm{H}$ & $\mathrm{M}$ & \\
\hline \multirow[t]{16}{*}{ Bloque Temático } & \multirow[t]{2}{*}{ Cronotipología } & Recuento & 201 & 50 & 251 \\
\hline & & $\%$ dentro de Género & $36,2 \%$ & $29,8 \%$ & $34,7 \%$ \\
\hline & \multirow[t]{2}{*}{ Arq. del Paisaje } & Recuento & 71 & 25 & 96 \\
\hline & & $\%$ dentro de Género & $12,8 \%$ & $14,9 \%$ & $13,3 \%$ \\
\hline & \multirow[t]{2}{*}{ Arqueometria } & Recuento & 13 & 9 & 22 \\
\hline & & $\%$ dentro de Género & $2,3 \%$ & $5,4 \%$ & $3,0 \%$ \\
\hline & \multirow[t]{2}{*}{ Arte } & Recuento & 61 & 17 & 78 \\
\hline & & $\%$ dentro de Género & $11,0 \%$ & $10,1 \%$ & $10,8 \%$ \\
\hline & \multirow[t]{2}{*}{ Datación Abs } & Recuento & 27 & 3 & 30 \\
\hline & & $\%$ dentro de Género & $4,9 \%$ & $1,8 \%$ & $4,1 \%$ \\
\hline & \multirow[t]{2}{*}{ Paleoambiente } & Recuento & 21 & 14 & 35 \\
\hline & & $\%$ dentro de Género & $3,8 \%$ & $8,3 \%$ & $4,8 \%$ \\
\hline & \multirow[t]{2}{*}{ Descriptivos/Revis } & Recuento & 99 & 34 & 133 \\
\hline & & $\%$ dentro de Género & $17,8 \%$ & $20,2 \%$ & $18,4 \%$ \\
\hline & \multirow[t]{2}{*}{ Otros } & Recuento & 62 & 16 & 78 \\
\hline & & $\%$ dentro de Género & $11,2 \%$ & $9,5 \%$ & $10,8 \%$ \\
\hline \multirow{2}{*}{\multicolumn{2}{|c|}{ Total }} & Recuento & 555 & 168 & 723 \\
\hline & & $\%$ dentro de Género & $100,0 \%$ & $100,0 \%$ & $100,0 \%$ \\
\hline
\end{tabular}

Tabla 2.- Tabla de contingencia con la distribución por género de los bloques temáticos. 
Pruebas de chi-cuadrado

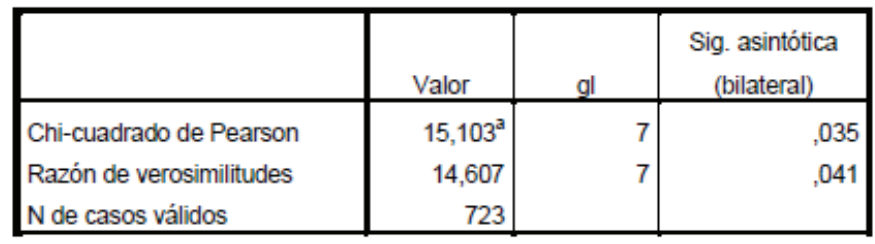

a. 0 casillas $(, 0 \%)$ tienen una frecuencia esperada inferior a 5 . La frecuencia mínima esperada es 5,11.

Tabla 3.- Resultado de la prueba de $C h i^{2}$ para la distribución por género de los bloques temáticos.

piamente el resultado de una colaboración activa en proyectos de investigación transfronteriza. Al margen de esta escasa colaboración directa cabe preguntarse en qué medida los autores portugueses y españoles se han interesando por difundir sus investigaciones en los ámbitos académicos del país vecino. Los autores afincados en España firman en un total de 523 trabajos; el $81.5 \%$ de esos trabajos aparecen en publicaciones con sede en España, el 15.3\% en publicaciones portuguesas y el 3.2\% restante en publicaciones británicas, francesas y belgas. Los autores afincados en Portugal firman casi exclusivamente en revistas o congresos con sede en su propio país ( $95 \%$ de un total de 322 trabajos); el porcentaje de firmas en publicaciones con sede en España es sólo del 4.7\%, mientras que una única firma aparece en medios con sede fuera de la Península (1 trabajo en una publicación francesa, el $0.3 \%$ ).

Terminaremos este apartado sobre autoría con un análisis del género. El 74.6\% (211) del total de 283 autores son hombres, mientras que las mujeres representan el 24.7\% (70). El 0.7\% restante corresponde a firmas colectivas (Grupo de Estudos Arqueologicos do Porto; Grupo de Arqueoloxía da Terra de Trasancos) que agrupan a miembros de ambos sexos. La introducción de la mujer en la investigación de la monumentalidad del noroeste es tardía, algo que puede hacerse extensivo al conjunto de la investigación arqueológica en España (Díaz-Andreu y Sanz Gallego 1994). La primera autora registrada en la muestra es María Pura Lorenzana, que publica un trabajo en 1930 junto con F. López Cuevillas y A. Fraguas Fraguas. Sólo tres mujeres más (Rosalía Polo en 1951, Sor Lucila González en 1953 y Margaret A. Smith en 1955) firman con anterioridad a 1970. A partir de esta fecha se incrementa el número de autoras, si bien los porcentajes respecto a los hombres se mantienen en niveles bajos hasta la década de 2000 (fig. 11).

Una de las cuestiones que nos interesaba analizar era si el modo de aproximación al objeto de estudio era similar entre los autores independientemente de su género. Un vistazo global al conjunto de datos daba, en efecto, la sensación de que la mujer estaba más presente en determinado tipo de temáticas. Para verificar y objetivar esta impresión planteamos un test estadístico que nos permitiese comparar la orientación principal de los trabajos respecto al sexo de los firmantes. Para eliminar el sesgo producido por la práctica ausencia de mujeres en fechas anteriores a 1970, este test se ha limitado al período 1970-2010. Los porcentajes de trabajos sobre Arqueología del Paisaje, Arqueometría, Paleoambiente y los trabajos de revisión son algo superiores entre las mujeres (Tabla 2). Entre los hombres predominan los estudios de Cronotipología, Arte, Datación absoluta y el conjunto de trabajos con otras temáticas minoritarias. Las mayores diferencias se dan en los estudios de cronotipología $(36.2 \%$ del total de firmas de los hombres, frente al $29.8 \%$ del total de firmas de las mujeres) y de paleoambiente $(8.3 \%$ del total de firmas de las mujeres, frente al 3.8\% del total de firmas de hombres). El test de $C h i^{2}$ de Pearson para el conjunto de estas observaciones (Tabla 3 ) confirma que el modo en que las mujeres y los hombres se aproximan al estudio de la monumentalidad del noroeste es diferente, y que estas diferencias son estadísticamente significativas (p-valor/sig. asintótica 0.035, inferior al umbral estadístico de 0.050).

\subsection{Análisis regional}

Este apartado tiene por objetivo analizar qué regiones han sido objeto de estudio preferente. La mayor parte de los trabajos $(574$, el $90.83 \%$ del total de 632) tratan un único contexto geográfico, pudiendo ser éste de diversa escala (un municipio; un ámbito provincial; un ámbito nacional; un ámbito regional; un ámbito transnacional - noroeste, península ibérica, fachada atlántica europea -). 31 trabajos (el $4.90 \%$ del total de 632 ) estudian dos contextos, 15 $(2.38 \%)$ estudian tres y los restantes $(12$, el $1.89 \%)$ estudian más de 4 (hasta un máximo de 10). Este 


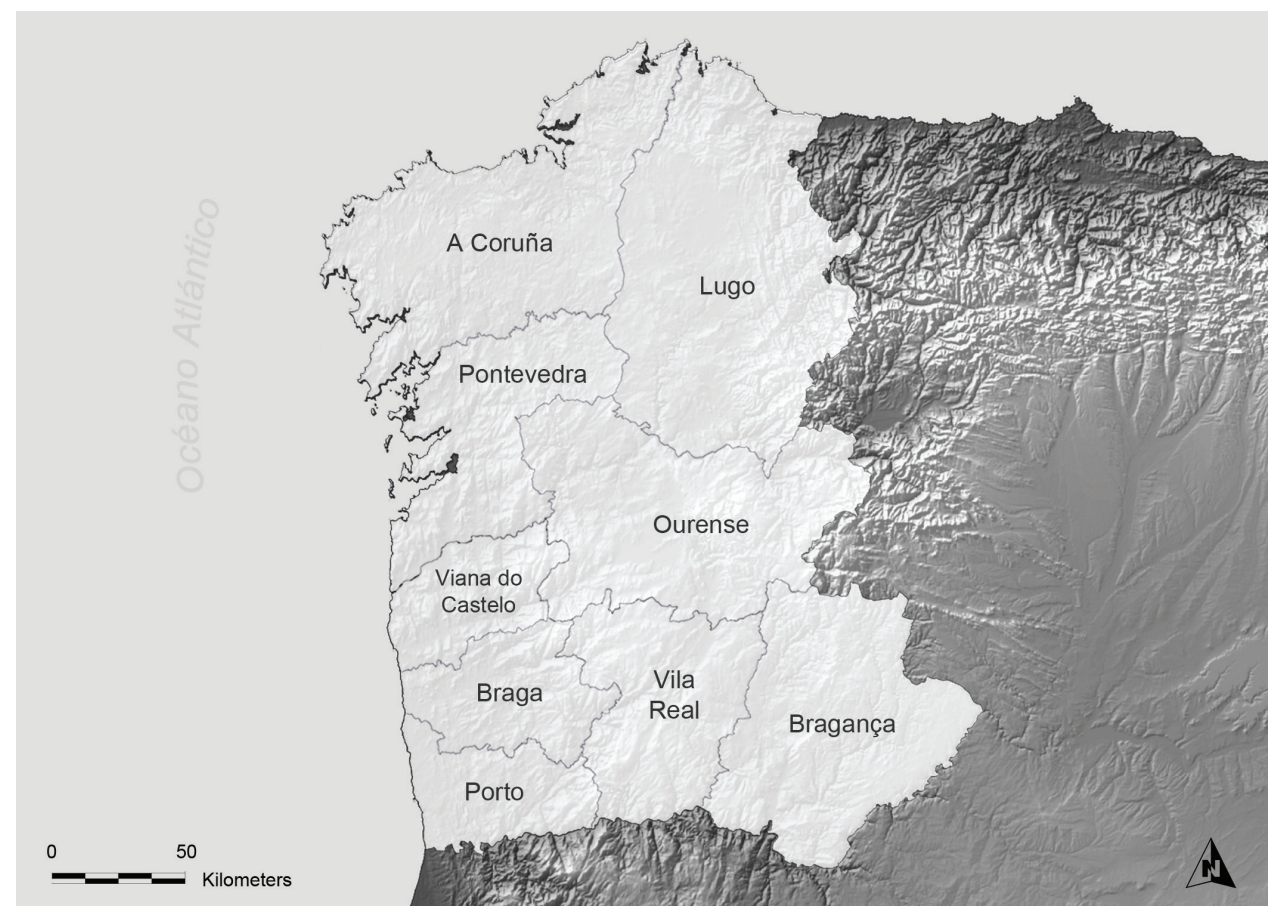

Fig. 12.- Divisiones administrativas actuales: escala provincial.

último bloque de trabajos suele no obstante coincidir con el análisis de subregiones de Galicia que agrupan a varios municipios, caso de la Baixa Limia (Ourense), Terra Chá (Lugo) o la península de Barbanza (A Coruña). Veamos a continuación con un poco más de detalle la diversificación geográfica en función de las distintas escalas de aproximación al territorio.

Varios trabajos hacen referencia a varias zonas, por lo que el número de localidades tratadas supera al número de artículos; el resultado es de 760 áreas estudiadas en los 632 trabajos de la muestra. La mitad de las zonas se ubican en Galicia (383, el 50.4\% del total de 760), mientras que las zonas localizadas en el norte de Portugal representan el 35.4\% (269). El 7\% (53) corresponde a áreas ubicadas a ambos lados de la frontera hispano-portuguesa tratadas de forma conjunta en los trabajos. El 2.9\% (22) se refiere a contextos atlánticos en los que se incluye algún tipo de discusión sobre el noroeste ibérico. Tres localizaciones $(0.4 \%)$ no han podido ser identificadas a partir de los datos publicados.

El análisis de las provincias más estudiadas ofrece datos interesantes sobre las áreas preferentes de investigación a escala intrarregional (fig. 12). Varios trabajos se centran en contextos generales (Fachada Atlántica, Península Ibérica, Noroeste, España, Portugal, Galicia, norte de Portugal) para los cuales no es posible definir un nivel de análisis regional más detallado; este tipo de localizaciones representa el $32.1 \%$ del total de 760 . Limitándonos al subconjunto de escala intrarregional (502 ocurrencias, el $67.9 \%$ restante) las provincias más estudiadas son las de A Coruña (objeto de estudio en 115 ocasiones), Pontevedra (81), Porto (80) y Lugo (78). Siguen las provincias de Vila Real (40), Ourense (38), Viana do Castelo (37), Braga (17) y Bragança (16).

Los estudios sobre el norte de Portugal (en especial sobre el distrito de Vila Real) son claramente predominantes hasta el segundo cuarto del siglo XX. A partir de ese momento se produce un aumento muy significativo en el estudio de las provincias gallegas (en un primer momento A Coruña y Lugo, hasta 1950, y a continuación Pontevedra hasta mediados de los años 1970). Con la expansión del número de trabajos a partir de 1980 se observa una mayor diversificación. Los estudios en el norte de Portugal resurgen, encabezados por los trabajos en el distrito de Porto. Los últimos años de la investigación recogidos en la muestra (2001-2010) se caracterizan por un predominio de estudios sobre las provincias de A Coruña y Pontevedra; el porcentaje de trabajos sobre Lugo se reduce de forma significativa (tendencia decreciente visible desde mediados del siglo XX), mientras que los de Pontevedra y Vila Real se incrementan ligeramente. En 


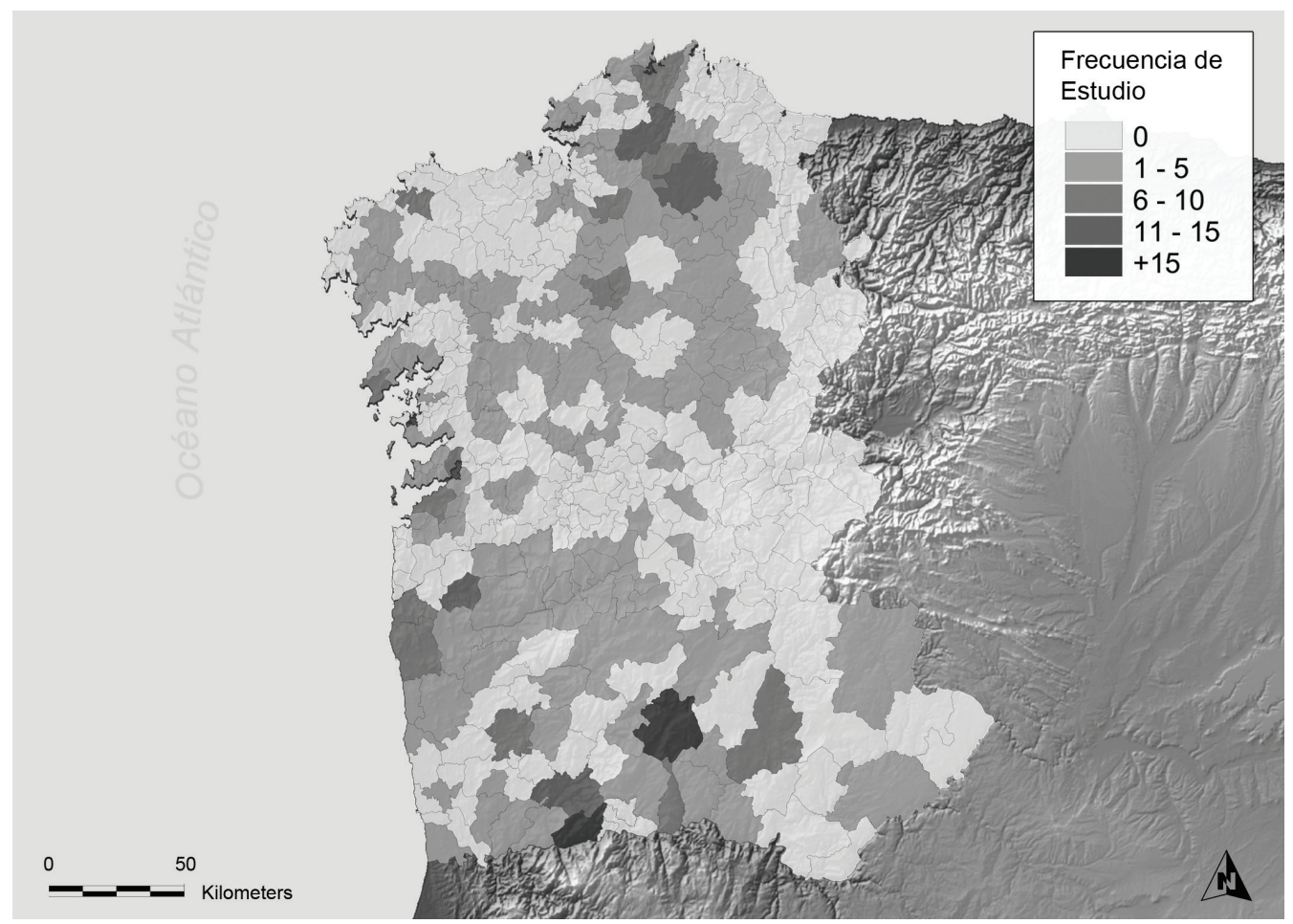

Fig.13.- Frecuencia de estudio de cada municipio.

este período no se registra ningún trabajo sobre las provincias de Ourense, Porto o Bragança.

El peso de los dos núcleos que más investigadores aportan (Santiago de Compostela y Porto, $v$. apartado Autoría) ha de ser tenido en cuenta a la hora de valorar el predominio de las investigaciones en la provincia de A Coruña y en la región de Porto. Sin embargo, este localismo no basta para explicar la representatividad de las áreas objeto de estudio. Por un lado, la importancia de conjuntos monumentales de otras provincias como Pontevedra y Lugo hace que investigadores externos a ellas se interesen por su estudio. Por otro lado, conjuntos como los de Aboboreira (Porto) o Barbanza (A Coruña) son lo suficientemente significativos como para haber atraído el interés de la investigación y haber sido objeto de un número importante de trabajos. El análisis de los municipios más estudiados corrobora esta impresión.

El municipio más frecuentemente estudiado es con 50 ocurrencias el de Baião (Porto). Le siguen Vila Pouca de Aguiar (16 ocurrencias; Vila Real), Amarante (13; Porto), As Pontes de García Rodríguez (12; A Coruña), Vilalba (12; Lugo), Paredes de Coura (11; Viana do Castelo), Guimarães (10; Braga) y Vigo (10; Pontevedra). El resto de localidades son objeto de estudio en menos de nueve ocasiones (fig. 13).

\section{Discusión}

\subsection{Una lectura histórica}

Tanto en Galicia como en el del Norte de Portugal el fenómeno de la monumentalidad se encuentra firmemente arraigado en la vida y en el imaginario colectivo de las sociedades de la región. Durante la Edad Media y la Edad Moderna los monumentos forman parte del paisaje como marcos territoriales (e.g. Martinón-Torres 2001). Al mismo tiempo aparecen leyendas y topónimos que los relacionan con el pueblo mítico de los 'Mouros'. Los primeros intentos de comprensión histórica del fenómeno comienzan a finales del siglo XVIII con la llegada de las corrientes de pensamiento racionalista. En Galicia destaca la figura de Fray Martín Sarmiento (1695-1772). A mediados del siglo XIX, tal y como ocurre en otras regiones, las aproximaciones de corte romanticista vinculan el fenómeno al mundo celta y hacen referencia a los ritos druídicos a él asociados. Se produce una tendencia a la elaboración de volúmenes sobre la historia local o regional, entre ellas varias Historias de Galicia. Autores como José Verea y Aguiar, José Villaamil, o Manuel Murguía refieren en sus trabajos el origen céltico de los monumentos. La Historia de 
Galicia de Murguía, de 1865, es sin duda uno de los trabajos más destacados. El recurso a la excavación arqueológica como método de obtención de información es por el contrario muy escaso en el noroeste si lo comparamos con otras regiones europeas. El portugués Francisco Martins Sarmento (1833-1899) destaca no obstante en este sentido. Aunque su labor se centra en el mundo castreño, Martins Sarmento llevó a cabo varias actuaciones sobre túmulos en Braga y Viana do Castelo. El Museo de la Sociedade Martins Sarmento, fundado en 1885 en Guimarães, recogió las colecciones resultantes de su actividad. El éxito de la Sociedade debe mucho al hecho de que se tratase de una iniciativa privada con fines de desarrollo local, frente a iniciativas estatales (como el Museu do Algarve instalado en Lisboa en 1880) de encaje mucho más problemático (Fabião 1999: 111-13).

Es aproximadamente en este punto en el que se sitúa el inicio de la muestra bibliométrica de nuestro trabajo. La producción científica denota que en estos momentos la actividad arqueológica es especialmente activa en áreas del norte de Portugal. Coincidiendo con los inicios de la revista O Archeólogo Português (1895), dirigida por José Leite de Vasconcellos, personajes como Henrique Botelho, José Brenha o Raphael Rodrigues exploran intensamente los monumentos de Vila Real. La preocupación por el conocimiento y la preservación del registro están claramente presentes (Rodrigues 1895: 346). La situación no escapa a los círculos científicos del momento, y algunos autores gallegos se quejan del poco interés que despierta el estudio de los túmulos en Galicia frente al desarrollo que éstos tienen en Portugal (e.g. Vázquez Núñez 1901). A partir de la década de 1920 la situación comienza a cambiar, algo que en la muestra queda reflejado por el inicio de los trabajos en las provincias de A Coruña y Ourense. Este punto de inflexión en la dinámica investigadora se oficializa entre 1922 y 1927 con la presencia de Hugo Obermaier y Luis Pericot en Galicia. El trabajo de ambos va a suponer un impulso importante para la introducción de metodologías y perspectivas de investigación que se estaban dando en otras áreas peninsulares (Armada-Pita 2008).

El predominio de los estudios de cronotipología que observamos en la muestra tiene un referente fundamental en el estudio de Galicia y norte de Portugal realizado por Georg Leisner en 1938. Si bien el volumen no presenta grandes novedades desde el punto de vista del inventario de monumentos, el análisis formal de las cámaras y la mención a las manifestaciones pictóricas asociadas marcaron las líneas a seguir dentro de un programa de trabajo positivista.
A pesar de estas líneas dominantes (impulsadas desde ámbitos externos como Madrid, Barcelona o Lisboa) figuras como la de Federico Maciñeira (1870-1943) constituyen un magnífico ejemplo de renovación 'desde dentro'. Ajeno a los ámbitos estrictamente académicos, Maciñeira destaca en el ámbito de la Arqueología por su rigor empírico, por su trabajo de campo y por su visión de la relación entre los monumentos y la geografía. Quizás no sea exagerado decir que sus análisis sobre el emplazamiento de los conjuntos de túmulos presentan ciertas concordancias con la futura Arqueología del Paisaje (e.g. Maciñeira 1935).

La colaboración entre investigadores se observa tanto a nivel interno (gallegos-portugueses) como externo a la región. El caso más evidente de esta movilidad lo tenemos en la figura de F. BouzaBrey, que visita Bretaña en 1929 y 1933. Aquí entra en contacto con Z. Le Rouzic (Bouza-Brey 1943) y con el registro megalítico de la región de Carnac, cuyo estudio y restauración se habían intensificado desde 1900 (López-Romero y Le Gall 2008: 69-70). La existencia de este tipo de redes, pese a ser esenciales y pese a su relativa intensidad (Martins 2010: 23-6), no se tradujo en una internacionalización de los trabajos publicados; la gran mayoría de los autores siguieron firmando en solitario y en medios regionales.

Además del descenso significativo en el número de trabajos en la década de 1930, la desaparición del gallego como idioma de publicación entre ese momento y mediados de la década de 1970 se explica por la reorganización completa del estado español como resultado de la Guerra Civil y la instauración de la dictadura franquista. A pesar de que el gallego había tenido poca representación en las décadas anteriores a 1930, la imposición del castellano como única lengua española dio al traste con la diversidad trilingüe del noroeste. La Guerra Civil puso igualmente fin a proyectos regionalistas de gran proyección como el Seminario de Estudos Galegos, creado en 1923 (Díaz-Andreu 1997: 407) y al que perteneció María Pura Lorenzana, la primera mujer en la muestra que publica sobre monumentalidad. En el caso de Portugal, donde frente al éxito de las iniciativas individuales las estructuras de investigación tuvieron problemas para consolidarse, se dio un ligero movimiento de renovación que supuso entre otras cosas la creación (1933) de la Junta Nacional de Escavações, Antiguidades e Numismática (Martins 2009: 29). A pesar de que el desarrollo de la disciplina estuvo limitado por el sistema autoritario del Estado Novo (1933-1974; Jorge y Jorge 1995: 247) algunos autores han subrayado el contraste entre la estabilidad política portuguesa desde los años 1920 y el 
conflicto interno que supuso la guerra en España (Fabião 1999: 107).

Tras la guerra la investigación retoma poco a poco su curso en Galicia. Se realizan varios trabajos de síntesis sobre el megalitismo gallego, destacando los de F. López Cuevillas (1955) que se ocupó también de las manifestaciones gráficas asociadas a los monumentos (1943). La actividad de éste y otros investigadores se realiza desde el Instituto de Estudios Gallegos Padre Sarmiento que desde los años 40 va a impulsar la investigación arqueológica en Galicia.

El conflicto personal e institucional entre A.A. Mendes Corrêa (Faculdade de Ciências da Universidade do Porto) y M. Heleno (Faculdade de Letras de Lisboa y conservador del Museu Etnológico) marcó una parte de la investigación en Portugal desde 1933 hasta finales de los años 1950. Ambos compartían no obstante un discurso de justificación nacionalista (Martins 2009) en el que el megalitismo (imperio megalítico, Fabião 1999: 121) jugaba un papel importante. La investigación prehistórica debe por lo demás a Corrêa algunas de las más significativas síntesis del momento (e.g. Corrêa 1947).

La fase de expansión en los estudios sobre monumentalidad se da únicamente a partir de 1970, algo que coincide en el tiempo con el final de los regímenes autoritarios en ambos países. La historiografía portuguesa ha acuñado el término renascimento para este momento, siendo la idade de ouro (segunda mitad del siglo XIX) y la idade das trevas (c. segundo y tercer cuarto del siglo $\mathrm{XX}$ ) los otros ejes de esta clasificación (Lemos 1987; $v$. Jorge y Jorge 1995). Aunque esta segmentación es algo reduccionista hay que reconocer que sus tres fases son fácilmente identificables dentro de nuestra muestra.

El papel de los museos y centros de investigación cobra gran relevancia a partir de estos momentos. La Universidade de Santiago de Compostela (con la creación de una Especialidad de Prehistoria y Arqueología, primera promoción de 1977-1978) y la Universidade do Porto van a resultar esenciales en la formación de arqueólogos. Se crean nuevos centros educativos como la Universidade do Minho con sede en Braga (1973), la Universidade Portucalense (Porto, 1986), la Universidade de Coruña (1989) o la Universidade de Vigo (1990). El incremento en el número de profesionales va a traducirse en un aumento en el número de investigaciones. En estos momentos se producen importantes actuaciones en conjuntos como Barbanza, Parxubeira o Bocelo, en Galicia, o la Serra da Aboboreira, en Portugal, al tiempo que se avanza en el conocimiento de la cultura material (Vázquez Varela 1979; Fábregas Valcárce 1991).
Se generalizan las excavaciones en área, lo que resultará esencial para la comprensión de la complejidad del registro asociado a los monumentos.

El número de publicaciones se incrementa notablemente, si bien por lo general se sigue privilegiando el ámbito regional (revistas como Espacio, Tiempo y Forma, o Arqueologia-Porto).

El papel jugado por los museos provinciales es igualmente destacado. En Galicia, los museos de A Coruña, Ourense, Pontevedra y el museo vigués Quiñones de León contribuyen al incremento de las actividades de prospección, llevándose a cabo los primeros estudios polínicos en contextos neolíticos (e.g. Aira Rodríguez y Guitián Ojea 1984). La introducción en los años 1980 y 1990 de aproximaciones de corte más típicamente procesual y, poco después, postprocesual, supusieron un cambio sustancial en el tratamiento epistemológico y metodológico de la monumentalidad. Los estudios paleoambientales se desarrollaron de forma progresiva, abarcando aspectos como las relaciones entre túmulos y medio geológico o el análisis de paleosuelos (e.g. Martínez-Cortizas y Moares Domínguez 1995).

La asunción de competencias autonómicas y la creación del Servicio de Arqueoloxia de la Dirección Xeral de Patrimonio son igualmente factores de crecimiento de la investigación en Galicia (Martínez Díaz 2002). La aplicación de las normativas en prevención de impacto arqueológico ha contribuido a ampliar el número y tipo de registro conocido. En Galicia los resultados de estas actuaciones se publican regularmente desde 2006 en forma de resúmenes (serie Actuacións Arqueolóxicas).

En los últimos años se han intensificado las investigaciones relacionadas con el estudio arqueométrico de los contextos monumentales en la región, en especial en lo relativo al estudio de la cerámica. Avances muy significativos se han producido igualmente en la contextualización cronológica del 'arte megalítico', así como en la consideración patrimonial y puesta en valor de los conjuntos tumulares.

\subsection{Una lectura transversal}

Más allá de esta lectura evolutiva de las investigaciones apoyada en el análisis bibliométrico nos interesa llevar a cabo una lectura más abstracta sobre el modo en que el estudio de la monumentalidad ha sido abordado en el noroeste.

Puede decirse que las posturas formalistas e historicistas han sido las predominantes. El análisis del 'objeto arqueológico' como fuente primaria de información ha dirigido gran parte de la investigación, acompañado y luego en parte sustituido por un concepto de 'yacimiento arqueológico' muy limitado por lo general al estudio formal de las cámaras. 
El problema de la focalización en el objeto ha sido que ésta se ha reducido fundamentalmente a una descripción y un encuadre cronológico del mismo, estando generalmente desprovista de profundidad interpretativa. Un ejemplo de esto lo tenemos en el uso de patrones cronotipológicos externos al noroeste para ubicar determinados elementos de la cultura material. Así, el empleo del concepto de Calcolítico ha generado un debate en el que las características sociales y económicas de las sociedades estudiadas han jugado un papel secundario frente a los aspectos formales y cronológicos que denotaba determinado tipo de objeto.

El estudio de los monumentos ha reproducido esta postura, haciendo del yacimiento un lugar central del discurso que necesitaba ser clasificado formal y cronológicamente. El análisis de las cámaras y corredores adquiere de este modo un innegable protagonismo en el que los modelos de referencia proceden inicialmente de áreas alejadas como el Alentejo o Andalucía. Sólo en momentos recientes este concepto de yacimiento se amplía con la toma de conciencia de la complejidad de las estructuras tumulares y con la puesta en perspectiva de éstas como elementos integrantes de un espacio geográfico y cultural igualmente complejo.

La adopción de una perspectiva de 'paisaje arqueológico' implica un importante punto de ruptura con las posturas formalistas. Sin dejar de lado el análisis de la cultura material y las arquitecturas, el yacimiento es considerado desde una perspectiva multidimensional en la que la interrelación entre sus distintos elementos y su contexto espacial son la base de su interpretación. Se lleva a cabo una revalorización de la estructura del túmulo como fuente de información, se introduce el análisis estratigráfico, se discute la existencia de túmulos sin cámaras ortostáticas, se analiza la frecuentación de los yacimientos en distintas épocas, se interpretan los patrones de localización de los túmulos en su entorno y contexto paleoambiental, se afronta el problema de la escasa visibilidad de los lugares de hábitat y su relación con los monumentos. Pese a que esta perspectiva se realiza en la región tanto desde postulados de corte procesual como postprocesual, cabe destacar la contribución de la aproximación estructuralista al establecimiento de un modelo interpretativo sobre la monumentalidad atlántica (Criado Boado 1989).

Si bien tanto la perspectiva formalista como la de paisaje encuentran su máxima representatividad en momentos concretos, ambas pueden identificarse a lo largo de la historia de la investigación de la región y coexisten a lo largo del tiempo. Así, la perspectiva de F. Maciñeira en la primera mitad del siglo XX contrasta con las aproximaciones tipolo- gicistas dominantes, al igual que trabajos de corte histórico-cultural perviven en las décadas de máximo desarrollo de la perspectiva de paisaje y en la actualidad.

Teniendo esto presente, ¿cuáles son las tendencias actuales en el estudio de la monumentalidad del noroeste y cuáles sus posibles puntos de desarrollo?

Aunque la muestra analizada nos ofrece pocos datos sobre las dinámicas de la investigación en este inicio del siglo XXI, la consideración de las tendencias más actuales en el estudio del fenómeno puede darnos algunas pautas de lectura de las mismas.

Si como acabamos de ver los conceptos de 'objeto arqueológico', 'yacimiento arqueológico' y 'paisaje arqueológico' han dominado el estudio de la monumentalidad en la región, en la última década se observa una tendencia a la consolidación de una perspectiva de 'patrimonio'. En la muestra analizada esta perspectiva se traduce en una serie de trabajos recientes sobre consolidación y puesta en valor entre los que destacan la conservación de pinturas y la musealización de yacimientos como Dombate o Forno dos Mouros. Muy lejos del concepto decimonónico de 'monumentos históricos' (Swenson 2006) es ésta una visión que radica en los cambios operados en torno a la década de 1990 (Criado Boado 1996: 16) y que hace hincapié en el valor socioeconómico, en la divulgación o en la multivocalidad como modos complementarios de aproximación y preservación de su objeto de estudio.

Frente a la multidisciplinaridad que caracterizó a varias de las intervenciones de 1980-1990, el reto de esta perspectiva ha de ser lograr la imbricación de todas las partes para alcanzar una auténtica mirada interdisciplinar al objeto de estudio. Esto supone superar algunas de las herencias más fuertemente arraigadas del paradigma histórico-cultural, poner al servicio de la interpretación global del fenómeno el conjunto de conocimientos y herramientas que se encuentran a nuestra disposición, y garantizar unas adecuadas medidas para su gestión sostenible. En el plano más estrictamente metodológico esta perspectiva implica la apertura disciplinar de los estudios sobre monumentalidad a otros ámbitos del conocimiento, así como la intensificación de la colaboración activa con especialistas procedentes de dichas áreas. Esto se hace especialmente necesario en el caso de la Arqueometría (e.g. Montero Ruiz et al. 2007). Algunas iniciativas han servido muy recientemente para dinamizar este tipo de estudios y su interpretación social en el noroeste ibérico (Prieto Martínez et al. 2008; Pétrequin et al. 2012). En esta línea, el empleo de métodos de datación absoluta alternativos al radiocarbono para la comprensión de los procesos constructivos y postdeposicionales 
relacionados con los monumentos está dando resultados en otras áreas de la fachada atlántica europea (Scarre 2011; v. López-Romero 2011).

En el plano de la implementación esta labor pasa igualmente por redefinir los contextos de estudio. Como ha quedado de manifiesto a lo largo del presente trabajo el fenómeno de la monumentalidad en el noroeste es esencialmente tumular y predomina en contextos de interior. Como también es sabido, el medio edafológico que predomina en dichos contextos no favorece la preservación de macrorestos de materia orgánica, con las implicaciones que esto tiene para la valoración de los aspectos funerarios, paleobiológicos, antropológicos e incluso genéticos vinculados a la monumentalidad. Es evidente que estos condicionantes de carácter tafonónico han impedido el conocimiento de estos aspectos en el noroeste frente a otras regiones como la Estremadura portuguesa, la Meseta o Andalucía; pero no es menos cierto que la búsqueda de contextos alternativos capaces de proporcionar esa información cualitativa deficitaria en la región no ha sido en absoluto explorada. El descubrimiento de nuevos monumentos en el pequeño islote de Guidoiro Areoso (Rey García y Vilaseco Vázquez 2012), en Galicia, o casos del norte de Portugal como la Mamoa de Chafé (Silva y Marques 1986) nos hacen reflexionar sobre la existencia de un contexto litoral para la monumentalidad que, aunque quizás minoritario, puede aportar datos enormemente relevantes en razón de su adecuación para la preservación de macrorestos orgánicos. Del mismo modo, las investigaciones sobre las ocupaciones en cueva han permitido identificar restos de enterramientos en Valdavara I (Becerreá, Lugo) y en Pala da Vella (Rubiá, Ourense) que cabe situar a finales del IV milenio BC (Vaquero Rodríguez et al. 2009; Fernández Rodríguez y Villar Quinteiro 2003); en el caso de la primera el hallazgo de dientes y falanges podría evidenciar, según los investigadores, un episodio de manipulación de los restos humanos, algo que encajaría con el modo de tratamiento de los individuos en contextos tumulares que conocemos para otras áreas peninsulares y atlánticas.

\section{Conclusión}

La investigación sobre la monumentalidad en el noroeste ibérico no escapa a lo que ha sido la historia de la disciplina a nivel peninsular, si bien presenta algunas líneas de fuerza propias que merecen ser tenidas en cuenta.

La producción científica reflejada a través del análisis bibliométrico marca una serie de pulsos claramente identificables. El inicial desarrollo de los trabajos en la región norte de Portugal da paso a un estancamiento a inicios del siglo XX, momento en el que la investigación en Galicia toma el relevo apoyada (entre otras cosas) por un mejor afianzamiento de las estructuras de investigación en España. Ambas regiones sufren el peso de los regímenes autoritarios que se dan en España y Portugal hasta la década de 1970; una de las consecuencias más visibles de esta fase es la desaparición del gallego como idioma de comunicación científica.

A pesar de la proximidad geográfica y de las distintas problemáticas comunes la investigación ha traspasado en pocas ocasiones las actuales fronteras administrativas. Es cierto que la relación entre investigadores gallegos y portugueses ha sido activa en distintas fases del período analizado, pero ésta ha sido más (in)formativa que realmente colaborativa. Escasean los trabajos en coautoría y de estudio transfronterizo de la región. Esta tendencia encuentra parte de su explicación en el relativo localismo de la investigación (sobre el concepto de localismo $v$. Rodríguez Alcalde et al. 1996, 56). Existe localismo puesto que muchos trabajos se centran en yacimientos o conjuntos muy concretos (limitados geográficamente a determinados municipios o comarcas), áreas aledañas como el oeste de Asturias apenas forman parte de las discusiones, se privilegian las revistas locales o regionales, y se observa un muy bajo índice de difusión internacional de la investigación. Este localismo es sin embargo relativo, puesto que existen toda una serie de trabajos de síntesis que denotan una voluntad de generalización del objeto estudiado; estas síntesis son de dos tipos: 'internas' (que tratan el fenómeno en una parte o en la totalidad del noroeste) o 'externas' (que tratan el contexto del noroeste en el marco de síntesis más amplias sobre la prehistoria peninsular, portuguesa, española o atlántica).

El desarrollo de los estudios de Prehistoria y Arqueología en la región a partir de 1980 y 1990 debe mucho a los estudios sobre monumentalidad. Los trabajos en áreas como Aboboreira, Barbanza o Bocelo fueron el vector de gran parte de la renovación teórico-metodológica que se operó en la región. La presencia de la mujer en la investigación (enormemente escasa hasta ese momento) se hizo entonces más patente. Como dato destacado se observa una diferencia en el tipo de temáticas abordadas por ambos sexos, algo que ha sido aquí modelizado con el estudio estadístico de la orientación de los trabajos.

Tras el descenso en la intensidad de la investigación que observamos a partir de la década de 2000 queda por ver si las líneas de desarrollo esbozadas al final del trabajo llegan finalmente a consolidarse. La situación socioeconómica y de investigación que atraviesan Portugal y España nos hace preguntarnos si no estaremos más bien entrando en una nova idade das trevas. 


\section{Agradecimientos}

Este trabajo es parte de la investigación realizada en el seno del Instituto de Ciencias del Patrimonio (Incipit) en el marco de un contrato del programa JAE-Doc del CSIC (2009-2012) cofinanciado por el Fondo Social Europeo. C. Le Gall (CReAAH-Université de Rennes1) y el Prof. C. Fabião (Universidade de Lisboa) proporcionaron acceso a distintas publicaciones. Gracias a F. Criado-Boado y A.C. González-García por compartir comentarios y experiencias.

\section{REFERENCIAS BibLIOGRÁFiCAS}

Abad Vidal, E; Aboal Fernández, R; Carballo Arceo, X; Casal García, R. (eds) (2000): Bibliografía arqueolóxica de Galicia (1980-1998). Asociación Profesional de Arqueólogos de Galicia, Deputación Provincial de Pontevedra.

Aira Rodríguez, M.J.; Guitián Ojea, F. (1984): Estudio polínico y edafológico de los yacimientos de Regueiriño y Fontenla (Península del Morrazo, Pontevedra). Pontevedra Arqueológica, I: 99-112.

Armada-Pita, X.-L. (2008): Arqueólogos en el finisterre: Obermaier, Pericot y la cátedra de Santiago de Compostela. Documentos inéditos para la Historia de la Arqueología (G. Mora, C. Papí, M. Ayarzagüena, eds.), Memorias de la Sociedad Española de Historia de la Arqueología 1. Sociedad Española de Historia de la Arqueología. Madrid: 197-212.

ARmadA-Pita, X.-L. (2009): Indicadores bibliométricos, visibilidad y calidad de revistas científicas: en torno a Revista d'Arqueologia de Ponent. Revista d'Arqueologia de Ponent, 19: 7-28.

Bello Diéguez, J.M.; Criado Boado, F.; Vázquez Varela, J.M. (1982): Aproximación a un modelo económicosocial del megalitismo del noroeste peninsular. Brigantium, 3: 33-39.

Bouza-Brey, F. (1943): Grabados rupestres serpentiformes en tierras de Lugo. Boletín de la Comisión Provincial de Monumentos Históricos y Artísticos de Lugo, 6: 134-138.

Cordero, T.; García-Sanjuán, L.; Hurtado, V.; Martín Ramírez, J.M.; Polvorinos del Río, A.; Taylor, R. (2006): La arqueometría de materiales cerámicos: una evaluación de la experiencia andaluza. Trabajos de Prehistoria, 63 (1): 9-35.

CorrêA, A.A.M. (1947): Histoire des recherches préhistoriques en Portugal. Trabalhos de Antropologia e Etnologia (Porto), 11: 115-170.

Criado Boado, F. (1989): Megalitos, espacio, pensamiento. Trabajos de Prehistoria, 46: 75-98.

Criado BoAdo, F. (1993): Visibilidad e interpretación del registro arqueológico. Trabajos de Prehistoria, 50: 3956.

Criado Boado, F. (1996): El futuro de la Arqueología, ¿la Arqueología del futuro?. Trabajos de Prehistoria, 53(1): 15-35.

Cruells, W. (1995): Aproximació bibliomètrica i índexs de Cota Zero (1985-1995). Cota Zero, 11: 100-122.

Cruz Berrocal, M., Goytre Samaniego, J., Leal Valladares, G.; López Domínguez, M. (1999): Crítica al estudio del Arte Levantino desde una perspectiva bibliométrica. Trabajos de Prehistoria, 56(1): 53-75.

DíAz-ANDReu, M. (1997): Nación e internacionalización. La arqueología en España en las tres primeras décadas del siglo XX. La cristalización del pasado: génesis y desarrollo del marco institucional de la Arqueología en España (G. Mora, M. Díaz-Andreu, eds.), Málaga: 403-416.

Díaz-Andreu, M.; Sanz Gallego, N. (1994): Women in Spanish Archaeology. Equity Issues for Women in Archaeology (M.C. Nelson, S.M. Nelson, A. Wylie, eds.), Archaeological Papers of the American Anthropological Association 5: 121-130.

FABIÃo, C. (1999): Um século de arqueologia em Portugal. Al-Madan, II série, 8: 104-126.

Fábregas Valcarce, R. (1991): Megalitismo del Noroeste de la Península Ibérica. Tipología y secuencia de los materiales líticos. UNED. Madrid.

Fernández Rodríguez, C.; Villar Quinteiro, R. (2003). Prospección y excavación de cuevas en la Cuenca del Sil (Rubiá, Ourense): La Pala da Vella. Brigantium, 14: 13-22.

García-Heras, M. (1997): Estudio bibliométrico de los trabajos de caracterización sobre materiales cerámicos arqueológicos en España: una valoración. Revista d'Arqueologia de Ponent, 7: 129-150. 
García Marín, A.; Rodríguez Alcalde, A.L.; San Millán Bujanda, M.J.; Vicente Bobadilla, G. de; Martínez Navarrete, M.I. (1997): ¿Nos pasamos de la raya?: la frontera hispanoportuguesa a través de las publicaciones de Prehistoria y Protohistoria. Trabajos de Prehistoria, 54(1): 35-56.

Jorge, V.O.; Jorge, S.O. (1995): Theoretical underpinnings of Portuguese Archaeology in the twentieth century. Theory in Archaeology: a world perspective (Ucko, P.J., ed.), London/New York, Routledge: 247-258.

LEISNER, G. (1938): Verbreitung und typologie der Galizisch-NordportugiesischenMegalithgräber. Marburg.

Lemos, F.S. (1987): As três idades da arqueologia portuguesa. Forum, 2: 5-11.

López Cuevillas, F. (1927): O ídolo cIlíndrico do museu arqueolóxico de Pontevedra e outros ídolos galegos. Arquivos do Seminario de Estudos Galegos, 1: 11-20.

López Cuevillas, F. (1955): Caracteres de la cultura megalítica del noroeste. III Congreso Nacional de Arqueología (Galicia 1953), Institución Fernando el Católico, Zaragoza: 261-272.

López-Romero, E. (2011): Datación por Luminescencia Ópticamente Estimulada de monumentos megalíticos: contexto y perspectivas. Exploring Time and Matter in Prehistoric Monuments: Absolute Chronology and Rare Rocks in European Megaliths (L. García-Sanjuán, C. Scarre, D. Wheatley, eds.), Menga Monográfico 1. Junta de Andalucía: 193-214.

López-Romero, E.; Le Gall, C. (2008): Le fonds documentaire de Geoffroy d'Ault du Mesnil (1842-1921) et la recherche préhistorique en France. Les Dossiers du Ce.R.A.A., 36: 65-72.

López-Romero, E.; Montero Ruiz, I. (2006): Archaeometry and the international evolution of studies on metallurgy: a bibliometric perspective. 34th International Symposium on Archaeometry, Institución Fernando el Católico-CSIC (3-7 mayo de 2004), Zaragoza: 195-200.

MACIÑEIRA, F. (1935): La distribución de las estaciones prehistóricas ortegalesas y sus características. Boletín de la Real Academia Gallega, 22(259): 169-181.

Martínez Cortizas, A.; Monés Domínguez, C. (1995): Edafología y Arqueología. Estudio de yacimientos arqueológicos al aire libre en Galicia. Xunta de Galicia, Santiago de Compostela.

Martínez Díaz, B. (2002): Veinte años de Arqueología en el Estado de las Autonomías. Historiografía de la Arqueología española. Las instituciones (S. Quero Castro, A. Pérez Navarro, coord) Museo de San Isidro, Serie Cursos y Conferencias 3, Madrid: 223-247.

Martins, A. C. (2009): Mendes Corrêa, Manuel Heleno and Portuguese Archaeology during the Estado Novo. Two names. Two cities. Two perspectives. One nationalism. Archaeologia Polona, 47: 155-178.

Martins, A. C. (2010): Iberian crossroads: archaeology and dictatorships. Bulletin of the History of Archaeology, 20(2): 21-31. doi:10.5334/bha.20204

MartinÓN-Torres, M. (2001): Los megalitos de término. Crónica del valor territorial de los monumentos megalíticos a partir de las fuentes escritas. Trabajos de Prehistoria, 58 (1): 95-108.

Montero Ruiz, I.; García-Heras, M.; López-Romero, E. (2007): Arqueometría: Cambios y Tendencias Actuales. Trabajos de Prehistoria, 64(1): 23-40.

Palomar, T.; García-Heras, M.; Villegas, M.A. (2009): Archaeological and historical glasses: A bibliometric study. Boletín de la Sociedad Española de Cerámica y Vidrio, 48(4): 187-194.

Pericot García. Ll. (1927): Los vasos campaniformes de la colección La Iglesia. Boletín de la Real Academia Gallega, 16(192): 283-291.

Pétrequin, P.; Cassen, S.; Klassen, L.; FÁBregas Valcárce, R. (2012): La circulation des haches carnacéennes en Europe occidentale. JADE. Grandes haches alpines du Néolithique européen, Ve au IVe millénaires av. J.-C. (P. Pétrequin, P., S. Cassen, M. Errera, L. Klassen, A. Sheridan, A.M. Pétrequin, eds) Les Cahiers de la MSHE Ledoux, Presses universitaires de Franche-Comté: 1015-1045.

Prieto Martínez, P.; Lantes SuÁrez, O; Martínez Cortizas, A. (2008): O campaniforme cordado de Forno dos Mouros (Toques, A Coruña). Cuadernos de Estudios Gallegos, 55(121): 31-51.

Rey García, J.M.; Vilaseco VÁzquez, X.I. (2012). Guidoiro Areoso. Megalithic cemetery and prehistoric settlement in the Ria de Arousa (Galicia, NW Spain). Environmental changes and human interaction along the western Atlantic edge. Mudanças ambientais e interação humanana fachada atlântica ocidental (A. Campar Almeida, A.M.S. Bettencourt, D. Moura, S. Monteiro-Rodrigues, M.I. Caetano Alves, eds) Coimbra, APEQ Associação Portuguesa para o Estudo do Quaternário: 243-258.

Rodrigues, R. (1895): Dolmens ou antas de Villa Pouca de Aguiar (2ªrtigo). O Arqueólogo Português, I série, 1(2): 346-352. 